\title{
8. \\ Von dem Krümmungs - Schwerpuncte ebener Curven.
}

(Von Hrn. Prof. Steiner zu Berlin.)

(Auszug aus einer am 5. April 1838 in der hiesigen Akad. d. Wissensch. gehaltenen Vorlesung.)

(Fortsetzung des Aufsatzes No. 3. im vorigen Hefte,)

\section{Von Figuren, die durch rollende Bewegung erzeugt werden.}

A. Wenn eine gegebene Figur $\mathfrak{B}$ auf einer festen Geraden $G$ rollt.

\section{s. XXVI.}

Rollt ein beliebiges convexes Vieleck $\mathfrak{B}$, z. B. das Fünfeck $\boldsymbol{A B C D E}$ (Fig. 6.) auf einer festen Geraden $G$, bis es sich ganz umgedreht hat, wobei seine Seiten alle nach - und nebeneinander auf die Gerade $\boldsymbol{G}$ zu liegen kommen und das Vieleck zuletzt wieder auf derselben Seite steht, wie anfangs, so dafs also die Strecke $\boldsymbol{A A}_{1}$ seinem Umfange gleich ist, so beschreibt jeder mit dem Vielecke fest verbunden-gedachte Punct $\boldsymbol{P}$ eine Linie $\boldsymbol{P P}_{1} \boldsymbol{P}_{2} \boldsymbol{P}_{3} \boldsymbol{P}_{4} \boldsymbol{P}_{5}$, die aus so vielen Kreisbogen zusammengesetzt ist, als das Vieleck Seiten hat. Und zwar haben diese Kreisbogen $\boldsymbol{P P}_{1}$, $\boldsymbol{P}_{1} \boldsymbol{P}_{2}, \ldots \ldots \boldsymbol{P}_{4} \boldsymbol{P}_{5}$ die Puncte $\boldsymbol{A}, \boldsymbol{B}_{1}, \boldsymbol{C}_{1}, \ldots \boldsymbol{E}_{1}$, in welchen die Ecken $\boldsymbol{A}, \boldsymbol{B}, \ldots \boldsymbol{E}$ des Vielecks $\mathfrak{B}$ auf die Gerade $\boldsymbol{G}$ treffen, zu Mittelpuncten, die Strahlen $a, b, c, d$, e, welche den Punct $P$ mit den Ecken $A, B, C, \ldots$ des Vielecks verbinden, zu Radien, und zu Centriwinkeln die Nebenwinkel $\boldsymbol{A}, \boldsymbol{B}, \boldsymbol{C}, \boldsymbol{D}, \boldsymbol{E}$ der an diesen Ecken gelegenen Winkel des Vielecks. Die Linie $\boldsymbol{P P}_{1} \boldsymbol{P}_{2} \ldots \boldsymbol{P}_{5}$ und die drei Geraden $\boldsymbol{A P}, \boldsymbol{A A}_{1}$ und $\boldsymbol{A}_{1} \boldsymbol{P}_{5}$ begrenzen eine Figur $\boldsymbol{A P P}_{1} \ldots \boldsymbol{P}_{5} A_{1}$, welche als aus folgenden $T$ Theileu zusammengesetzt betrachtet werden kann: 1) Aus einer Reihe von (n) Dreiecken $A P_{1} B_{1}, B_{1} P_{2} C_{1}, \ldots E_{1} P_{5} A_{1}$, welche beziehlich den Dreiecken $A P B, B P C, \ldots E P A$ gleich sind, in die das gegebene Vieleck $\mathfrak{B}$ durch die Strahlen $a, b, \ldots e$ zerfällt wird, so dafs die Inhaltssumme jener Dreiecke dem Inhalte dieses Vielecks gleich ist; und 2) aus einer gleichen Anzahl von Kreissectoren, deren Mittelpuncte, Radien und Centriwinkel bereits näher angegeben worder sind. Diese Figur $\boldsymbol{A P P}_{1} \boldsymbol{P}_{2} \ldots \boldsymbol{P}_{5} \boldsymbol{A}_{1}$ soll fortan "von dem Puncte $P$ beschrieben" heifsen. Bezeichnen wir sie

Crelle's Journal d. M. Bd. XXI. Hft. 2. 14 
oder ihren Inhalt mit $W$, so erhellet aus dem Gesagten, dals :

63. $\boldsymbol{W}=\mathfrak{V}+\frac{1}{2} a^{2} \boldsymbol{A}+\frac{1}{2} b^{2} \boldsymbol{B}+\frac{1}{2} c^{2} \boldsymbol{C}+\ldots=\mathfrak{B}+\frac{1}{2} \Sigma\left(a^{2} \boldsymbol{A}\right)$, wobei $A, B, C, \ldots$ die genannten Nebenwinkel des Vielecks $\mathfrak{B}$, in Zahlen ausgedrückt, sind.

Nach (\$. VII.) lärst sich die vorstehende Gleichung in folgende umwandeln :

$$
\text { 64. } \begin{aligned}
W & =\mathfrak{B}+\frac{1}{2} a_{1}^{2} A+\frac{1}{2} b_{1}^{2} B+\frac{1}{2} c_{1}^{2} C+\ldots .+\frac{1}{2} s^{2}(A+B+C+\ldots . .), \\
& =\mathfrak{B}+\frac{1}{2} \Sigma\left(a_{1}^{2} A\right)+\frac{1}{2} s^{2} \Sigma(A),
\end{aligned}
$$

wo $a_{1}, b_{1}, c_{1}, \ldots . s$ die Strahlen sind, welche einen in Rücksicht des

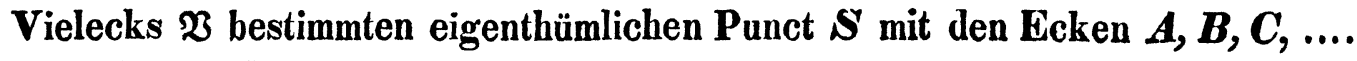
desselben und mit dem Puncte $\boldsymbol{P}$ verbinden.

Bemerkt man, dafs nach (\$. XVII. 32.):

so folgt (64):

$$
\text { 65. } \Sigma(A)=A+B+C+\ldots=2 \pi \text {, }
$$

$$
\text { 66. } W=\mathfrak{B}+\frac{1}{2} \Sigma\left(a_{1}^{2} A\right)+\pi s^{2},
$$

und daher für den Inhalt $w$ der von $\operatorname{dem}$ Puncte $S$ beschriebenen Figur, für welchen $s=0$ ist :

woraus in Verbindung mit (66) endlich folgt:

$$
\text { 67. } w=\mathfrak{B}+\frac{1}{2} \Sigma\left(a_{1}^{2} A\right),
$$

68. $W=w+\pi s^{2}$.

Aus allem zusammen ergeben sich folgende Sätze :

a) „Rollt ein beliebiges convexes Vieleck $\mathfrak{B}$ in einer Ebene auf einer festen Geraden $G$, bis es sich ganz umgedreht hat, so giebt es einen eigenthümlichen Punct $\mathbf{S}$, der unter allen mit dem Vieleck fest verbundenen Puncten $\boldsymbol{P}$ die dem Inhalte nach kleinste Figur $w$ beschreibt. Dieser ausgezeichnete Punct $\boldsymbol{S}$ ist der Schwerpunct der Ecken des gegebenen Vieleckes $\mathfrak{B}$, wenn denselben die respectiven Nebenwinkel des Vieleckes als Coefficienten zugeordnet werden."

b) „Jeder andere Punct $\boldsymbol{P}$ beschreibt eine Figur, deren Inhalt $\boldsymbol{W}$ gerade um diejenige Kreisfläche grö/ser ist als der Inhalt jener kleinsten Figur w, welche den Abstand s des Punctes $\mathbf{P}$ von $\mathbf{S}$ zum Radius hat." So dafs also :

c) ,Alle Puncte $\boldsymbol{P}$, welche in einer Kreislinie liegen, die $\boldsymbol{S}$ zum Mittelpuncte hat, Figuren $W$ von gleichem Inhalte beschreiben; und auch umgekehrt: Je ein System von Puncten $P$, welche Figuren $\boldsymbol{W}$ von gleichem 
Inhalie beschreiben, liegt in einem Kreise, dessen Mittelpunct der Schwerpunct $\mathbf{S}$ ist."

Dafs bei einem regelmäfsigen Vieleck $\mathfrak{B}$ der hier in Rede stehende Schwerpunct $\boldsymbol{S}$ mit dem Mittelpuncte des Vielecks zusammenfallen mufs, ist einleuchtend. Auch in andern besondern Fällen lärst sich dieser Schwerpunct $\boldsymbol{S}$ leicht angeben, oder geometrisch construiren, wie z. B. namentlich in dem Falle, wo die Nebenwinkel des Vielecks $\mathfrak{B}$ unter einander commensurabel sind. Beim Dreieck, Viereck etc. ergeben sich in dieser Hinsicht einige interessante specielle Sätze.

\section{s. XXVII.}

Der Inhalt der Figur $\boldsymbol{W}$ kann unter Beibehaltung seiner Bestandtheile auch in anderer Form oder durch eine andere Figur $\mathfrak{B}$ dargestellt werden, wobei es nicht nöthig ist, das Vieleck $\mathfrak{B}$ auf der Geraden $\boldsymbol{G}$ rollen zu lassen. Nämlich die in der Figur $W$ vorkommenden Kreissectoren (Fig. 6.) können unmittelbar an das Vieleck $\mathfrak{B}$ angeschlossen und zwar in seinen Nebenwinkeln $A, B, C, \ldots$ beschrieben werden, wie z. B. in (Fig. \%.), wo die Kreisbogen $\mathfrak{A P}_{1}, \mathfrak{B}_{\mathfrak{B}_{1}}, \mathfrak{S S}_{1} \ldots$ aus den Ecken $\boldsymbol{A}, \boldsymbol{B}, \boldsymbol{C}, \ldots$ mit den Radien $a, b, c, \ldots$ beschrieben sind. Auf diese Weise hat offenbar die Figur $\mathfrak{A}_{1} \mathfrak{B B}_{1} \mathfrak{E} \mathfrak{E}_{1} \mathfrak{D} \mathfrak{D}_{1} \mathfrak{E} \mathfrak{E}_{1}=\mathfrak{W}$ gleichen Inhalt mit jener Figur $\mathscr{W}$, welche der Punct $\boldsymbol{P}$ beim Rollen des Vielecks $\mathfrak{B}$ auf der Geraden $G$ (Fig. 6.) beschreibt. Da die Kreissectoren sich auf zwei verschiedene Arten so an das Vieleck $\mathfrak{B}$ antragen lassen, dafs sie alle nach einer Richtung um dasselbe herumliegen (je nachdem man die Nebenwinkel des Vielecks durch Verlängerung der Seiten nach der einen oder andern Richtung hin entstehen lälst), so giebt es auf diese Weise zwei verschiedene Figuren $\mathfrak{B}$ und $\mathfrak{B}_{1}$, die aber nothwendig gleichen IIIhalt haben.

Hiernach ist klar, dafs die oben ( $\$$. XXVI.) für die Figuren $W$ und $w$ entwickelten Formeln und Sätze auf gleiche Weise auch für die Figuren $\mathfrak{B}$ und $\mathfrak{w}$ Statt finden müssen, wo nämlich $\mathfrak{w}$ dem Schwerpuncte $S$ entspricht und mit $w$ gleichen Inbalt hat. Daher bat man :

$$
\begin{aligned}
& \text { 69. } \mathfrak{W}-\mathfrak{B}=\mathfrak{B}_{1}-\mathfrak{B}=\frac{1}{2} \Sigma\left(a_{1}^{2} \boldsymbol{A}\right)+\pi s^{2}, \\
& \text { 70. } \mathfrak{w}-\mathfrak{B}=\mathfrak{w}_{1}-\mathfrak{B}=\frac{1}{2} \Sigma\left(a_{1}^{2} \boldsymbol{A}\right), \\
& \text { 71. } \mathfrak{B}-\mathfrak{w}=\mathfrak{B}_{1}-\mathfrak{w}_{1}=\pi s^{2},
\end{aligned}
$$

und daraus folgende Sätze: 
a) „Zieht man aus den Ecken $A, B, C, \ldots$ eines beliebigen convexen Vielecks $\mathfrak{B}$ nach irgend einem in seiner Ebene liegenden Puncte $\boldsymbol{P}$ Strahlen $a, b, c, \ldots$ und beschreibt mit diesen, als Radien, in den respectiven Nebenwinkeln $\boldsymbol{A}, \boldsymbol{B}, \boldsymbol{C}, \ldots$ des Vielecks $\mathfrak{B}$ Kreissectoren, so ist die Inhaltssumme (WB- $\mathfrak{B})$ dieser Kreissectoren dann ein Minimum $(\mathfrak{w}-\mathfrak{B})$, wenn der Punct $\boldsymbol{P}$ mit demjenigen Puncte $\boldsymbol{S}$ zusammenfällt, welcher der Schwerpunct der Ecken des Vielecks $\mathfrak{B}$ ist, insofern denselben Coefficienten zugehören, die sich wie die respectiven Nebenwinkel verhalten."

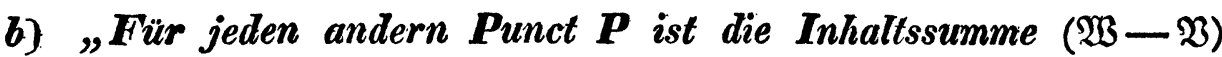
der Kreissectoren um diejenige Kreisfläche $\pi s^{2}$, welche den Abstand $s$ des $\boldsymbol{P}$ unctes $\boldsymbol{P}$ von $\boldsymbol{S}$ zum Radius hat, grö/ser als die dem Schwerpuncte $\mathbf{S}$ entsprechende Summe $(\mathfrak{w}-\mathfrak{B})$."

c) Puncten $\boldsymbol{P}$, die in einem Kreise liegen, dessen Mittelpunct $\boldsymbol{S}$

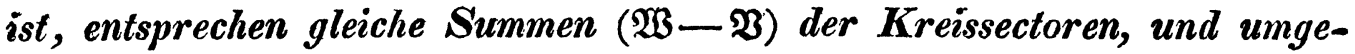
kehrt: alle Puncte $\boldsymbol{P}$, für welche die Inhaltssumme der Kreissectoren die nümliche ist, liegen in einem und demselben Kreise, dessen Mittelpunct $\mathbf{S}$ ist."

\section{S. XXVIII.}

Lärst man das bisher betrachtete Vieleck $\mathfrak{B}$ in eine Curve $\mathfrak{B}$ übergehen, wie oben ( $\$ X X$.), so müssen die aufgestellten Gleichungen und Sätze (S. XXVI. und XXVII.) auch für diesen Grenzfall noch Statt finden. Die übrigen mit betrachteten Figuren $\boldsymbol{W}$ und $\mathfrak{B}$ erhalten aber dadurch ebenfalls andere Formen, so wie der beschriebene Schwerpunet $\boldsymbol{S}$ eine characteristische Eigenschaft. Nämlich es treten folgende Aenderungen ein.

1) Roltt die geschlossene und convexe Curve $\mathfrak{B}$ auf der Geraden $\boldsymbol{G}$ (Fig. 6.), so ist die von jedem (mit der Curve $\mathfrak{B}$ fest verbundenen) Puncte $\boldsymbol{P}$ beschriebene Linie $\boldsymbol{P P}_{1} \ldots \boldsymbol{P}_{n}$, die früher aus Kreisbogen zusammengesetzt war, nun irgend eine bestimmte Curve $\boldsymbol{P P}_{n}$ (oder besteht aus unendlich vielen unendlich kleinen Kreisbogen). Die von dem Puncte $\boldsymbol{P}$ beschriebene Figur $\boldsymbol{W}$ ist das von der Curve $\boldsymbol{P P}_{n}$ und den drei Geraden $A P, P_{n} A_{1}$ und $A_{1} A_{1}$ eingeschlossene Viereck $\operatorname{APP}_{n} A_{1}$, wo, wie früher, die beiden ersten Geraden $\boldsymbol{A P}$ und $\boldsymbol{A}_{1} \boldsymbol{P}_{n}$ gleich und parallel und die dritte $\boldsymbol{A} \boldsymbol{A}_{1}$ dem Umfange der rollenden Curve $\mathfrak{B}$ gleich ist. 
2) Nach der in (\$. XXVII.) beschriehenen und in (Fig. 7.) dargestellten Construction der Figur $\mathfrak{W}$ folgt leicht, dafs für den gegenwärtigen Fall ihr Umfang in irgend eine bestimmte Curve $\mathfrak{W}$ übergeht. Denn da für diesen Fall die Nebenwinkel und die Seiten des Vielecks $\mathfrak{Z}$ alle unendlich klein werden, und die letztern in die Tangenten der Curve $\mathfrak{B}$ übergehen, so werden also auch die Kreisbogen $\mathfrak{A P}_{1}, \mathfrak{B P}_{1}, \mathfrak{E} \mathfrak{S}_{1}, \ldots$ sowohl als die Strecken $\mathfrak{A}_{\mathfrak{1}} \mathfrak{B}, \mathfrak{B}_{\mathfrak{l}} \mathfrak{S}, \mathfrak{C}_{1} \mathfrak{D}, \ldots$ alle unendlich klein, und daher müssen je drei auf einander folgende Puncte, wie z. B. $\mathfrak{A}, \mathfrak{A}_{1}$ und $\mathfrak{B}$ unendlich nahe bei einander liegen, so dafs also die genannte Curve $\mathfrak{B}$ schlechthin als Ort der Puncte $\mathfrak{A}, \mathfrak{B}, \mathfrak{E}, \ldots$ angesehen werden kann. D. h. wird auf jeder Tangente $A \mathfrak{A}$ der gegebenen Curve $\mathfrak{B}$ der ihrem Berührungspuncte $\boldsymbol{A}$ entsprechende Strahl $\boldsymbol{A P}=a$ abgetragen, wird $\boldsymbol{A} \mathfrak{A}=a$ gerrommen, so ist der Ort des Endpunctes $\mathfrak{A}$ der Tangente irgend eine bestimmte Curve $\mathfrak{W}$, welche die früher betrachtete Figur $\mathfrak{W}$ repräsentirt. Der Strahl $a$ kann aber von dem Berührungspuncte $\boldsymbol{A}$ aus nach zwei sich entgegengesetzten Richtungen hin auf der Tangente $\boldsymbol{A} \mathfrak{A}$ abgetragen werden. Daher entstehen durch das angegebene Verfahren zwei Figuren $\mathfrak{W}$ und $\mathfrak{W}_{1}$, welche zwar im Allgemeinen der Form nach von einander verschieden, aber stets von gleichem Inhalte sind, so dafs immer $\mathfrak{W}=\mathfrak{W}_{1}$.

3) Da der eigenthümliche Punct $\mathbb{S}$ beim Vieleck $\mathfrak{B}$ durch dessen Nebenwinkel $\boldsymbol{A}, \boldsymbol{B}, \boldsymbol{C}, \ldots$ bestimmt wird (\$. XXVI.), diese Winkel aber bei der Curve $\mathfrak{B}$, - wo sie unendlich klein sind - sich verhalten, wie die respectiven Krümmungen dieser Curve, oder wie die ungekehrten Werthe der respectiven Krümmungshalbmesser (\$. XXI.); so folgt (\$. XIII.): „da/s im gegenwärtigen Falle der eigenthümliche Punct $\mathbf{S}$ der nümliche ist, welcher oben (\$. XXII.) Krümmungs-Schwerpunct der Curve $\mathfrak{B}$ genannt wurde."

Wenn gleich hier die Winkel $\boldsymbol{A}, \boldsymbol{B}, \boldsymbol{C}, \ldots$ einzeln alle unendlich klein werden, so bleibt doch offenbar ihre Summe die nämliche, wie früher (\$. XXVI. 65.), also $\Sigma(\boldsymbol{A})=2 \pi$; und auch der Ausdruck $\frac{1}{2} s^{2} \Sigma(\boldsymbol{A})$ behält seinen frühern Werth $=\pi s^{2}$. Demnach finden für die eben beschriebenen Figuren $\mathfrak{B}, \mathfrak{B}, \boldsymbol{W}$ ganz dieselben Gleichungen Statt, wie oben (5. XXVI. und XXVII.), nämlich :

$$
\begin{array}{ll}
\text { 78. } & W=\mathfrak{W}=\mathfrak{B}+\frac{1}{2} \Sigma\left(a^{2} A\right), \\
\text { 73. } & W=\mathfrak{W}=\mathfrak{B}+\frac{1}{2} \Sigma\left(a_{1}^{2} A\right)+\pi s^{2}, \\
\text { 74. } & w=\mathfrak{W}=\mathfrak{B}+\frac{1}{2} \Sigma\left(a_{1}^{2} A\right),
\end{array}
$$




$$
\begin{aligned}
& \text { 75. } W=\mathfrak{B}=w+\pi s^{2}=\mathfrak{w}+\pi s^{2}, \\
& \text { 76. } \mathfrak{W}=\mathfrak{B}_{1} \text { und } \mathfrak{w}=\mathfrak{w}_{1},
\end{aligned}
$$

77. $(\mathfrak{B}-\mathfrak{B})=\left(\mathfrak{W B}_{1}-\mathfrak{B}\right)=(\mathfrak{W}-\mathfrak{B})+\pi s^{2}=\left(\mathfrak{w}_{1}-\mathfrak{B}\right)+\pi s^{2}$.

Die Vergleichung dieser Formeln mit denjenigen in (\$. XXIII.) insofern für alle dieselbe Curve $\mathfrak{B}$ zu Grunde gelegt und bemerkt wird, dafs für unendlich kleine Winkel $\sin 2 A=2 \sin A=2 A$ und also $\Sigma\left(a^{2} \sin 2 A\right)=2 \Sigma\left(a^{2} A\right)$ ist, - führt zu folgendem interessanten Resultate :

78. $W=\mathfrak{B}=2 V$ und $\mathfrak{w}=w=2 v$.

Aus allem diesem ergeben sich folgende Sätze:

a) „Rollt eine beliebige geschlossene und überall convexe Curve $\mathfrak{B}$ in ihrer Ebene auf einer festen Geraden $G$, bis sie sich ganz umgedreht hat, so beschreibt jeder mit ihr fest verbunden gedachte $P$ unct $P$ eine Figur $W$ (gemischtliniges Viereck), deren Inhalt von der Lage des Punctes $\boldsymbol{P}$ abhängt. Die vom Krümmungs - Schwerpuncte $\boldsymbol{S}$ der gegebenen Curve $\mathfrak{B}$ beschriebene Fägur hat unter allen den kleinsten Inhalt $w$. Für jeden andern Punct $\boldsymbol{P}$ ist der Inhalt der von ihm beschriebenen Figur $\boldsymbol{W}$ grö/ser, als dieses Minimum w, und zwar um diejenige KreisAlüche $\pi s^{2}$ grö/ser, welche den Abstand $s$ des Punctes $P$ vom KrümmungsSchwerpuncte $S$ zum Radius hat (75). Alle Puncte $P$ also, die in einem um den Schwerpunct $\mathbf{S}$ gezogenen Kreise liegen, beschreiben Figuren $W$ von gleichem Inhalte; und auch umgekehrt."

b) Werden aus irgend einem Puncte $\boldsymbol{P}$ in der Ebene einer beliebigen geschlossenen und convexen Curve $\mathfrak{B}$ Strahlen $a, b, c, \ldots$ nach allen Puncten $A, B, C, \ldots$ der Curve gezogen, und wird aus jedem Puncte der zugehörige Strahl auf die anliegende Tangente der Curve (nach einerlei Richtung) abgetragen, so bilden die Endpuncte $\mathfrak{A}, \mathfrak{B}, \mathfrak{S}, \ldots$. der Tangenten eine geschlossene Curve $\mathfrak{2}$. Unter allen Curven $\mathfrak{1 B}$, die auf solche Weise entstehen können, hat diejenige den kleinsten Inhalt $\mathfrak{w}$, welche dem Krümmungs - Schwerpuncte $\mathbf{S}$ der gegebenen Curve entspricht. Für jeden andern Punct $\boldsymbol{P}$ hat die entstehende Curve einen Inhalt $\mathfrak{B}$, der jenes Minimum $\mathfrak{w}$ um diejenige Kreisfäche $\pi s^{2}$ übertrifft, welche den Abstand $s$ des Puncts $P$ vom Schwerpuncte $\boldsymbol{S}$ zum Radius hat. Also entsprechen Puncten $\boldsymbol{P}$, die in einem um $\boldsymbol{S}$ (als Mittelpunct) gezogenen Kreise liegen, Curven $\mathfrak{B}$ von gleichem Inhalte; und auch umgekehrt." Feruer: „Je nachdem die Strahlen $a, b, c, \ldots$ in der einen oder der 
8. Steiner, von dem Krümmungs-Schwerpuncte ebener Curven.

andern Richtung auf die Tangenten der Curve $\mathfrak{B}$ abgetragen werden, entstehen für den nümlichen Punct $\boldsymbol{P}$ (S) zwei verschiedene Curven $\mathfrak{B}$ und $\mathfrak{W}_{1}$ ( $\mathfrak{w}$ und $\mathfrak{w}_{1}$ ), welche aber gleichen Inhalt haben (76)." Und weiter : „die Räume $(\mathfrak{W}-\mathfrak{B})$, $\left(\mathfrak{W}_{1}-\mathfrak{B}\right)$, welche die Curven $\mathfrak{B}$ und $\mathfrak{B}$, $\mathfrak{B}$ und $\mathfrak{W}_{1}$ zwischen sich abschlie/sen, sind für jeden Punct $\boldsymbol{P}$ einander gleich, und bleiben für alle Puncte $\boldsymbol{P}$, die in gleicher Entfernung s vom Krümmungs - Schwerpuncte $\mathbf{S}$ liegen, constant. Diese Räume haben den Ikleinsten Inhalt $\left(\mathfrak{w}-\mathfrak{B} ; \mathfrak{w}_{1}-\mathfrak{B}\right)$, wenn sie dem Puncte $\mathbf{S}$ entsprechen; für jeden anderen Punct $\boldsymbol{P}$ sind sie um die Kreisfläche $\pi s^{2}$, welche den

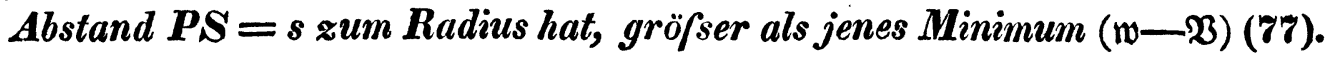

c) Betrachtet man dieselbe Curve $\mathfrak{Y}$ und denselben Punct $\boldsymbol{P}$ in Rücksicht auf beide vorigen Sätze (a) und (b), so hat die vom Puncte $P$ nach dem Satze (a) beschriebene Figur $W$ mit der ihm im Sinne des Satzes (b) entsprechenden Figur $\mathfrak{W}$ oder $\mathfrak{W}_{1}$ stets gleichen Inhalt, so dafs immer $\boldsymbol{W}=\mathfrak{W}=\mathfrak{W}_{1}$." Und ferner :

d) „Jede von den beiden Figuren $W$ oder $\mathfrak{Y B}$ hat gerade doppelt so gro/sen Inhalt, als die demselben Puncte $\boldsymbol{P}$ in Bezug auf dieselbe gegebene Curve $\mathfrak{B}$ entsprechende Fu/spuncten-Curve $V$ (78)," Oder ausführlicher :

a. Rollt die gegebene Curve $\mathfrak{B}$ auf einer festen Geraden $\boldsymbol{G}$, so beschreibt jeder mit ihr fest verbunden gedachte Punct $\boldsymbol{P}$ eine Figur $\boldsymbol{W}$, deren Inhalt gerade doppelt so gro/s ist, als derjenige der FurspunctenCurve $\boldsymbol{V}$, die dem nümlichen Puncte $\boldsymbol{P}$ in Bezug auf die nümliche gegebene Curve $\mathfrak{B}$ entspricht"; und:

ß. Bewegt sich ein veränderliches gleichschenkliges Dreieck $\boldsymbol{P A} \mathfrak{A}$ unter der Bedingung, da/s seine Spitze $\boldsymbol{A}$ die gegebene Curve $\mathfrak{B}$ durch-

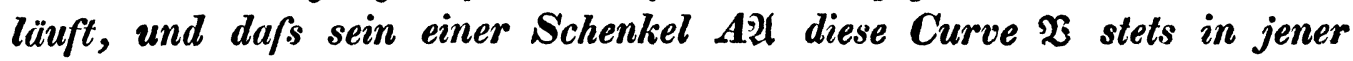
Spitze A tangirt, während die dem Schenkel A $\mathfrak{A}$ gegenüberliegende Ecke in einem und demselben Puncte $\boldsymbol{P}$ fest bleibt, so beschreiben die dritte Ecke $\mathfrak{A}$ des Dreieckes und der Fu/spunct $\boldsymbol{A}_{1}$ des aus der festen Ecke $\boldsymbol{P}$ auf den Schenkel A $\mathfrak{A}$ gefällten Perpendikels zwei Curven $\mathfrak{B}$ und $V$, von denen die erste $\mathfrak{B}$ jedesmal doppelt so gro/sen Inhalt hat, als die zweite V." 


\section{§. XXIX.}

\section{Besondere Fälle.}

Die vorstehenden allgemeinen Resultate, - bei welchen die gegebene Curve $\mathfrak{B}$, mit Ausnahme der Bedingung geschlossen und überall convex zu sein, eine ganz beliebige, ihre Gleichung z. B. algebraisch oder transcendent sein kann, und wobei eben so die Gleichungen der erzeugten Curven $\boldsymbol{V}, \boldsymbol{W}, \mathfrak{W}$ und $\mathfrak{B}_{1}$ nicht in Betracht kommen, die, wie leicht zu ermessen, sowohl von der Gleichung der gegebenen Curve $\mathfrak{B}$, als unter sich sehr verschieden sein können, - umfassen unter andern folgende sehr specielle Sätze :

$\alpha$. Wenn die gegebene Curve $\mathfrak{B}$ ein Kreis ist.

Rollt der Kreis $\mathfrak{B}$, dessen Radius $=r$, auf der festen Geraden $\boldsymbol{G}$, so beschreibt jeder mit ihm verbundene Punct $\boldsymbol{P}$ eine gewöhuliche $\mathrm{Cy}$ kloide $W$, - eine gemeine, gestreckte oder verkürzte, je nachdem beziehlich $\boldsymbol{P}$ auf der Kreislinie innerhalb oder aufserhalb derselben liegt, und zufolge (\$. XXVIII. 78. u. \$. XXIV. 50.) ist:

$$
\text { 79. } W=2 \pi r^{2}+\pi s^{2} \text {, }
$$

d. h. „der Inhalt jeder gewöhnlichen Cykloide $W$ ist so gro/s, als die Summe der doppelten Fläche des Erzeugungskreises $\mathfrak{B}$ und der Fläche desjenigen Kreises, der mit jenem concentrisch ist und durch den beschreibenden Punct $\boldsymbol{P}$ geht."

Ist insbesondere $s=r$, oder liegt der beschreibende Punct $P$ auf Jer Kreislinie $\mathfrak{B}$, so ist :

$$
\text { 80. } W_{1}=3 \pi r^{2} \text {, }
$$

d. h. "der Inhalt der gemeinen Cykloide ist dreimal so gro/s, als die Flüche des Erzeugungskreises;" ein allgemein bekannter Satz.

Wenn ferner $s=0$, also wenn $\boldsymbol{P}$ mit dem Mittelpuncte $\boldsymbol{S}$ des Kreises $\mathfrak{B}$ zusammenfällt, so ist :

$$
\text { 81. } v=2 \pi r^{2}
$$

was auch daraus erhellt, dafs in diesem Falle $v$ ein Rechteck ist, dessen Seiten bexiehlich dem Radius $r$ und dem Umfange $2 \pi r$ des Erzeugungskreises $\mathfrak{D}$ gleich sind.

Diesen drei Fällen entsprechend hat man (\$. XXVIII.):

$$
\begin{aligned}
& \text { 82. } \mathfrak{W}=2 \pi r^{2}+\pi s^{2}, \\
& \text { 83. } \mathfrak{W}_{1}=3 \pi r^{2}, \\
& \text { 84. } \mathfrak{w}=2 \pi r^{2},
\end{aligned}
$$


d. h. „den nümlichen Inhalt, wie die dem Puncte $\boldsymbol{P}$ entsprechende $\boldsymbol{C y}$ kloide $W$, hat diejenige Curve $\mathfrak{W}$, welche der Ort des Endpunctes $\mathfrak{A}$ aller Tangenten A der aus ihrem Berührungspuncte $\boldsymbol{A}$ nach dem festen Pole $\boldsymbol{P}$ gehende Strahl $\boldsymbol{P A}=$ a abgetragen uird."

Die Curve $\mathfrak{w}$ ist hier ein mit dem gegehenen $\mathfrak{B}$ concentriseher Kreis, dessen Radius $=r \sqrt{ } 2$; was leicht zu sehen ist.

Auch der Inbalt der Ringe, die zwischen der Curve $\mathfrak{B}$ und den Kreise $\mathfrak{B}$ liegen, lärst sich hier genau angeben, nämlich er ist:

85. $\mathfrak{W}-\mathfrak{B}=\pi r^{2}+\pi s^{2} ; \mathfrak{B} \mathfrak{B}^{1}-\mathfrak{B}=2 r^{2} \pi ; \mathfrak{w}-\mathfrak{B}=\pi r^{2}$. Im zweiten Falle $\mathfrak{W}^{1}-\mathfrak{B}$, findet kein eigentlicher Ring Statt, sondern ein sichelförmiger Raum (Mond), dessen Spitzen jedoch im Puncte $\boldsymbol{P}$ an einander stofsen.

Anmerkung. Bei der verkürzten Cykloide entsteht, wenn z. B. der Punct $\boldsymbol{P}$ in dem durch den anfänglichen Berührungspunet $\boldsymbol{A}$ gehenden Durchmesser des Kreises $\mathfrak{B}$ und oberhalb dieses letztern und der Basis $\boldsymbol{G}$ liegt, wie (Fig. 8.), eine Schleife $\boldsymbol{Q} \boldsymbol{Q}_{1}$, indem die Cykloide im Puncte $\boldsymbol{Q}$ sich selbst schneidet. Alsdann besteht ihr Inhalt, d. i. $W$, aus den zwei Räumen:

oder aus den drei Stücken :

$$
\operatorname{APQP}_{1} A_{1} A+Q R Q_{1} T Q,
$$

\section{$A P R A+A_{1} T P_{1} A_{1}+R Q_{1} T R$.}

In allen analogen Fällen, die Curve $\mathfrak{B}$ mag sein, welche man will, ist der Inhalt der Figur $W$ auf gleiche Weise zu bestimmen.

Zieht man die Gerade $\boldsymbol{P P}_{1}$, welche die Cykloide in den Puncten $\boldsymbol{P}$ und $\boldsymbol{P}_{1}$ berührt, so entsteht der Arbelos $\boldsymbol{P Q P}_{1} \boldsymbol{P}$, dessen Inhalt mit den der Schleife $Q R Q_{1} T Q$ immer einen leicht angeblichen Unterschied macht. Nämlich dieser Unterschied ist stets, demjenigen zwischen dem Rechtecke $A_{P P_{1}} A_{1} A$ und der Figur $W$ gleich. Oder wird $B P=x$, also $s=r+x$ gesetzt, so ist :

Arbel. $\left(\boldsymbol{P Q P} P_{1} P\right)-$ Schleife $\left(Q R Q_{1} T Q\right)=A P P_{1} A_{1} A-W=\pi\left(2 r s-s^{2}\right)$ $=\pi\left(r^{2}-x^{2}\right)$,

d. h. ,der Unterschied zwischen dem Inhalt des Arbelos PQP $_{1}$ und dem der Schleife $Q^{Q} Q_{1}$ ist auch gleich dem Unterschiede zwischen der Fläche des rollenden Kreises und der Fläche desjenigen Kreises, dessen $\boldsymbol{R} a-$ dius $x=s-r$ ist."

Crelle's Journal d. M. Bd. XXI. Hft. 2 , 
Ist also $x=r$, d. h. $s=2 r$, so ist auch $P Q P_{1}=Q Q_{1}$ oder: der Arbelos hat gerade gleichen Inhalt mit der Schleife.

$\beta$. Wenn die gegebene Curve $\mathfrak{V}$ eine Ellipse ist.

Aus (\$. XXVIII. 78. u. \$. XXIV. 55.) folgt

86. $W=\pi\left(a^{2}+b^{2}+s^{2}\right)$;

d. h. „rollt eine Ellipse $\mathfrak{B}$ in ihrer Ebene auf der festen Geraden $G$, bis sie sich ganz umgedreht hat, so beschreibt jeder mit ihr fest verbundene $\boldsymbol{P}$ unct $\boldsymbol{P}$ eine Figur $\boldsymbol{W}$, deren Inhalt gleich ist der Summe dreier Kreisfächen, welche beziehlich die halben Axen a und b der Ellipse und den Abstand s des Punctes $\boldsymbol{P}$ von ihrem Mittelpuncte $\mathbf{S}$ zu Radien haben."

Liegt insbesondere der beschreibende Punct $\boldsymbol{P}^{\mathbf{1}}$ in der mit der Ellipse concentrischen und durch ihre Brennpuncte gehenden Kreislinie, ist also $s^{2}=a^{2}-b^{2}$, so ist

\section{7. $W^{1}=2 \pi a^{2}$;}

d. h. ,der Inhalt der von dem $P_{u n c t e} \boldsymbol{P}^{1}$ beschriebenen Figur $W^{1}$ ist gerade doppelt so gro/s, als die Kreisfläche, welche die gro/se Axe 2 a der Ellipse $\mathfrak{B}$ zum Durchmesser hat."

Dem Mittelpuncte $\boldsymbol{S}$ der Ellipse entspricht

88. $w=\pi\left(a^{2}+b^{2}\right)=2 \pi g^{2}$;

d. h. , die von dem Mittelpuncte $\boldsymbol{S}$ der Ellipse beschriebene Figur hat einen Inhalt w, der so gro/s ist, als die Summe zweier Kreisfächen, welche die Axen der Ellipse $\mathfrak{B}$ zu Durchmessern haben; oder doppelt so grofs, als die Kreisfläche, welche einen der gleichen conjugirten Durchmesser der Ellipse zum Durchmesser hat. (\$. XXIV. B.)

Die vorstehenden drei Formeln (86., 87. u. 88.) stellen zugleich auch die Inhalte der respective entsprechenden Curven $\mathfrak{W}, \mathfrak{B}^{1}, \mathfrak{w}$ dar, eben so wie oben beim Kreise $\left(\alpha_{\text {. }}\right.$ ). Für die zwischen diesen Curven und der Ellipse $\mathfrak{B}$ liegenden Räume oder Ringe hat man:

$$
\begin{aligned}
& \text { 89. } \mathfrak{W}-\mathfrak{B}= \pi\left(a^{2}+b^{2}-a b-s^{2}\right) ; \mathfrak{B}^{1}-\mathfrak{B}=a \pi(2 a-b) ; \\
& \mathfrak{w}-\mathfrak{B}=\pi\left(a^{2}+b^{2}-a b\right) .
\end{aligned}
$$

B. Wenn eine Figur $\mathfrak{B}$ auf einer andern festen Figur $\mathfrak{U}$ rollt.

\section{XXX.}

Wenn in einer Ebene eín beliebiges convexes Vieleck $\mathfrak{B}$, z. B. $A B C D$ (Fig. 9.) auf der Aufsenseite eines andern festen convexen Viel- 
ecks $\mathfrak{U}=\mathfrak{D}_{1} \mathfrak{A B} \mathfrak{S} \mathfrak{D} \mathfrak{A}_{1}$ (welches auch blofs eine aus Geraden zusammengesetzte gebrochene Linie sein kann), mit welchem es nach der Reihe gleiche Seiten hat, so lange rollt (wobei je ein Paar gleiche Seiten auf einander zu liegen kommen), bis es wieder mit der nämlichen Seite $(\boldsymbol{D} A)$, wie anfangs, auf der Basis $\mathfrak{U}$ aufliegt, z. B. bis es in die Lage von $A_{1} B_{1} C_{1} D_{1}$ (= $A B C D$ ) gelangt : so beschreibt jeder mit dem rollenden Vielecke $\mathfrak{B}$ fest verbundene Punct $P$ irgend eine Figur $W=\boldsymbol{P P}_{1} \ldots$ $\boldsymbol{P}_{4} \mathfrak{A} \mathfrak{1} \mathfrak{D} \mathfrak{B P} \mathcal{P}$, welche (wie oben $\$$ XXVI.) aus so vielen Dreiecken und aus so vielen Kreissectoren zusammengesetzt ist, als das rollende Vieleck $\boldsymbol{V}$ Ecken hat. Die Dreiecke sind beziehlich denen gleich, in welche das Vieleck $\mathfrak{Y}$ durch die aus seinen Ecken $\boldsymbol{A}, \boldsymbol{B}, \boldsymbol{C}, \boldsymbol{D}$ nach dem Puncte $\boldsymbol{P}$ gezogenen Strahlen $a, b, c, \ldots$ zerlegt wird; also ist ihre Summe gleich dem Inhalte dieses Vielecks $\mathfrak{Y}$. Die Kreissectoren haben beziehlich die nämlichen Strahlen $\boldsymbol{a}, \boldsymbol{b}, \boldsymbol{c}, \ldots$ zu Radien, die Ecken $\mathfrak{A}, \mathfrak{B}, \mathfrak{E}, \ldots$ des Vielecks $\mathfrak{U}$ zu Mittelpuncten, und zu Centriwinkeln die Summen der entsprechenden Nebenwinkel beider Vielecke $\mathfrak{B}$ und $\mathfrak{U}$. Werden also, wie früher, die Nebenwinkel des Vielecks $\mathfrak{B}$ durch $\boldsymbol{A}, \boldsymbol{B}, \boldsymbol{C}, \ldots$, diejenigen des Vielecks $\mathfrak{U}$ durch $\mathfrak{H}, \mathfrak{B}, \mathfrak{C}, \ldots$ bezeichnet, so ist zufolge des Gesagten :

90. $W=\mathfrak{B}+\frac{1}{2} a^{2}(A+\mathfrak{O})+\frac{1}{2} b^{2}(B+\mathfrak{B})+\frac{1}{2} c^{2}(C+\mathfrak{S})+\ldots$

$$
=\mathfrak{B}+\frac{1}{2} \Sigma\left[a^{2}(\boldsymbol{A}+\mathfrak{A})\right] \text {. }
$$

Aus der Uebereinstimmung dieser Gleichung mit jener obigen (\$. XXVI. 63.) erkennt man sogleich, dafs auch für die gegenwärtige Betrachtung analoge Gesetze Statt finden, wie dort. Nämlich: wird der Schwerpunct der Ecken $\boldsymbol{A}, \boldsymbol{B}, \boldsymbol{C}, \ldots$ des Vielecks $\mathfrak{Y}$, wenn denselben die Coefficienten $(\boldsymbol{A}+\mathfrak{P}),(\boldsymbol{B}+\mathfrak{B}),(\boldsymbol{C}+\mathfrak{E}), \ldots$ zugeordnet sind, durch $\mathfrak{S}$ und werden seine Abstände von den Ecken $\boldsymbol{A}, \boldsymbol{B}, \boldsymbol{C}, \ldots$ des Vielecks $\mathfrak{B}$ und von dem Puncte $\boldsymbol{P}$ beziehlich durch $a_{1}, b_{1}, c_{1}, \ldots$ und $\mathfrak{s}$ bezeichnet, so läfst sich die vorstehende Gleichung (90) in folgende verwandeln (\$. VII. u. XXVI.):

$$
\text { 91. } \boldsymbol{W}=\mathfrak{B}+\frac{1}{2} \Sigma\left[a_{1}^{2}(\boldsymbol{A}+\mathfrak{A})\right]+\frac{1}{2} \mathfrak{s}^{2} \Sigma(\boldsymbol{A}+\mathfrak{A}) \text {, }
$$

oder, da nach (\$. XXVI. 65.) $\Sigma(A)=2 \pi$ ist, so hat man, wenn $\mathfrak{A}+\mathfrak{B}$ $+\mathfrak{S}+\ldots . .=\mathfrak{q}$ gesetzt wird,

$$
\text { 92. } W=\mathfrak{V}+\frac{1}{2} \Sigma\left[a_{1}^{2}(A+\mathfrak{A})\right]+\frac{1}{2} \mathfrak{b}^{2}(2 \pi+\mathfrak{q}) \text {, }
$$

wobei $\mathfrak{q}$ in der Figur (9) dem Winkel $\mathfrak{M}, \mathfrak{N}$ gleich ist, unter welehem die auf die erste und die letzte Seite $\left(\mathfrak{D}_{1} \mathfrak{A}\right.$ und $\left.\mathfrak{D} \mathfrak{A}_{1}\right)$ von $\mathfrak{U}$ errichteten Perpendikel $\mathfrak{M}$, und $\mathfrak{N}$, sich schneiden. 
Für die von dem Schwerpuncte $\mathcal{S}$ beschriebene Figur $w$ hat man demnach :

und daher folgt weiter :

$$
\text { 93. } w=\mathfrak{B}+\frac{1}{2} \Sigma\left[a_{1}^{2}(\boldsymbol{A}+\mathfrak{Q})\right] \text {, }
$$

$$
\text { 94. } W=w+\frac{1}{2} \mathfrak{s}^{2}(2 \pi+\mathfrak{q}) \text {. }
$$

Diese Gleichung enthält folgenden Satz:

"Wenn in einer Ebene ein beliebiges convexes Vieleck $\mathfrak{B}$ auf der Aufsenseite eines beliebigen festen convexen Vielecks $\mathfrak{U}$, mit dem es respective gleiche Seiten hat, so lange rollt, bis es wieder mit der anfänglichen Seite auf demselben aufliegt, so beschreibt jeder mit ihm fest verbundene Punct $\boldsymbol{P}$ eine Figur $\boldsymbol{W}$, deren Inhalt ein Minimum $=w$ wird, wenn der beschreibende Punct $\boldsymbol{P}$ mit dem Schwerpuncte $\mathfrak{S}$ der Ecken des Vielecks $\mathfrak{B}$ zusammenfält, insofern denselben die Summen der entsprechenden Nebenwinkel beider Vielecke $\mathfrak{B}$ und $\mathfrak{U}$ als Coefficienten zugehören. Alle Puncte $\boldsymbol{P}$, welche gleichweit von diesem Schwerpuncte $\mathfrak{S}$ abstehen, beschreiben Figuren $W$ von gleichem Inhalte, und auch umgeliehrt; und zwar ist für jeden Punct $\boldsymbol{P}$ der Inhalt $\boldsymbol{W}$ gerade um denjenigen Kreissector, der den Abstand $\mathfrak{s}$ zwischen $\boldsymbol{P}$ und $\mathfrak{S}$ zum Radius und die Summe $(2 \pi+\mathfrak{q})$ aller jener Nebenwinkel zum Centriwinkel hat, grö/ser als jenes genannte Minimum w."

\section{\$. XXXI.}

Vornehmlich zum Behufe späterer Betrachtungen mag über das Vorstehende noch Folgendes bemerkt werden :

Es ist klar, dafs der Schwerpunet $\mathbb{S}$ sowohl direct, als auf folgendem Wege gefunden werden kann. Man suche für die Ecken $\boldsymbol{A}, \boldsymbol{B}, \boldsymbol{C}, \ldots$ des Vielecks $\mathfrak{B}$ zwei $S$ chwerpuncte $\boldsymbol{S}$ und $\boldsymbol{S}_{1}$, indem man für den ersten $\boldsymbol{S}$ die Nebenwinkel des Vielecks $\mathfrak{B}$ selbst, für den zweiten $\boldsymbol{S}_{\mathbf{1}}$ die respectiven Nebenwinkel $\mathfrak{A}, \mathfrak{B}, \mathfrak{C}, \ldots$ des Vielecks $\mathfrak{U}$ als zugehörige Coefficienten ansieht. Nimmt man alsdann in der Geraden $\boldsymbol{S S}_{\mathbf{1}}$ denjenigen Punct 5 , der sie so theilt, dafs :

95. $S \mathfrak{S}: \mathbb{S}_{1} \mathfrak{S}=\mathfrak{q}: 2 \pi$,

so ist derselbe offenbar der verlangte Schwerpunct $\mathbf{S}$. - Sind insbesondere die Nebenwiukel eines jeden Vielecks unter sich gleich, so falleu die drei Puncte $\mathbb{S}, \boldsymbol{S}_{1}$ und $\mathfrak{S}$ zusammen. Dasselbe kann aber auch unter andera Bedingungen eintreffen. 
Ferner kann der Inhall der Figur $W$ unter anderer Form, nämlich durch zwei Figuren $\mathfrak{B}$ und $\mathfrak{T}$ dargestellt werden. Denn wird der obige Ausdruck für $W$ wie folgt zerlegt (90):

$$
\text { 96. } W=\mathfrak{B}+\frac{1}{2} \Sigma\left(a^{2} A\right)+\frac{1}{2} \Sigma\left(a^{2} \mathfrak{F}\right)=\mathfrak{B}+\mathfrak{F},
$$

and einzeln gesetzt:

$$
\text { 97. } \mathfrak{B}+\frac{1}{2} \Sigma\left(a^{2} A\right)=\mathfrak{W} ; \quad \frac{1}{2} \Sigma\left(a^{2} \mathfrak{A}\right)=\mathfrak{T},
$$

so kann man sich unter $\mathfrak{W}$ die nämliche Figur denken, welche bereits oben ( $\$$ XXVII.) construirt wordeu; $\mathfrak{i}$ aber soll diejenige Figur sein, welche durch die gesammten Kreissectoren gebildet wird, die in den Nebenwinkeln $\mathfrak{A}, \mathfrak{B}, \mathfrak{C}, \ldots$ des Vielecks $\mathfrak{U}$ mit den Strahlen $a, b, c, \ldots$ als Radien und zwar unter der Bedingung beschrieben werden, dafs alle Sectoren nach einerlei Richtung hin liegen; was wie bei $\mathfrak{B}$, auf zwei verschiedene Arten geschehen kann.

Ueber den Inhalt der Figur $\mathfrak{B}$ sind die wesentlichsten Relationen am genannten Orte aufgestellt; nämlich er wird ein Minimum $=\mathfrak{w}$, wenn sie dem $\mathbf{S e h w e r p u n c t e} \boldsymbol{S}$ entspricht; aufserdem ist für jeden andern Punct $\boldsymbol{P}$ :

$$
\text { 98. } \mathfrak{W}=\mathfrak{w}+\pi s^{2},
$$

wo $s$ den Abstand des Puncts $\boldsymbol{P}$ von $\boldsymbol{S}$ bezeichnet.

Wird die Figur $\mathfrak{I}$ für sich betrachtet, so folgt ähnlicherweise, dafs ihr Inhalt dann ein Minimum $=t$ wird, wenn sie dem oben genannten Schwerpuncte $S_{1}$ entspricht, und dafs für jeden andern Punct $P$

$$
\text { 99. } \mathfrak{z}=\mathrm{t}+\frac{1}{2} \mathfrak{q} \cdot s_{1}^{2}
$$

ist, wo $s_{1}=P S_{1}$ und $\mathfrak{q}=\mathfrak{A}+\mathfrak{B}+\mathfrak{C}+\ldots$, (S. XXX.).

Demnach hat man (96):

100. $W=\mathfrak{B}+\mathfrak{T}=\mathfrak{w}+\pi s^{2}+t+\frac{1}{2} \mathfrak{q} \cdot s_{1}^{*}$.

Die Formel (99) enthält folgenden Salz:

„Der Inhalt der Figur $\mathfrak{T}$ ist dann ein Minimum $=\mathrm{t}$, wenn sie dem Schwerpuncte $\boldsymbol{S}_{1}$ entspricht; beliebigen Puncten $\boldsymbol{P}$, welche gleichweit vom Schwerpuncte $\boldsymbol{S}_{\mathbf{1}}$ abstehen, entsprechen Figuren $\mathfrak{I}$ von gleichem Inhalte, und auch amgekehrt; und zwar ist der jedesmalige Inhalt gerade um denjenigen Kreissector grö/ser als jenes Minimum $t$, welcher den Abstand $s_{1}$ der Puncte $P$ von $S_{1}$ zum Radius und den constanten Winkel q zum Centriwinkel hat." 


\section{s. XXXII.}

Bleiben alle Voraussetzungen über die Vielecke $\mathfrak{B}$ und $\mathfrak{U}$ die nämlichen, wie oben (\$S. XXX.), nur dafs $\mathfrak{B}$, statt auf der Aufsenseite, jetzt auf der innern, concaven Seite von $\mathfrak{U}$ rollen soll; so sind dabei im Allgemeinen drei Fälle zu unterscheiden, nämlich entweder sind:

a) Die Nebenwinkel $\boldsymbol{A}, \boldsymbol{B}, \boldsymbol{C}, \ldots$ des Vielecks $\mathfrak{B}$ alle grörser, als die ihnen entsprechenden Nebenwinkel $\mathfrak{A}, \mathfrak{B}, \mathfrak{\mathfrak { C }}, \ldots$ von $\mathfrak{U}$; oder :

$\beta)$ die erstern alle kleiner als die letztern; oder endlich

$\gamma$ ) die Nebenwinkel $\boldsymbol{A}, \boldsymbol{B}, \boldsymbol{C}, \ldots$ von $\mathfrak{B}$ theils kleiner, theils grö [ser (oder theils, wenn man will, auch gleich) als die Nebenwinkel $\mathfrak{A}, \mathfrak{B}, \mathfrak{C}, \ldots$. von $\mathfrak{U}$.

Im ersten Fall - der am leichtesten darzustellen ist und am meisten mit dem früheren übereinstimnt, daher hier auch allein berücksichtigt werden soll - beschreibt jeder mit dem Vieleck $\mathfrak{B}$ fest verbundene Punct $\boldsymbol{P}$ irgend eine Figur $\boldsymbol{W}$, welche auf analoge Weise, wie oben, aus Dreiecken, deren Summe $=\mathfrak{V}$ ist, und aus Kreissectoren besteht, deren Radien $a, b, c, \ldots$, deren Centriwinkel dagegen $A-\mathfrak{A}, \boldsymbol{B}-\mathfrak{B}$, $C-\mathfrak{E}, \ldots .$. sind; so dafs also hier:

101. $W=\mathfrak{B}+\frac{1}{2} \Sigma\left[a^{2}(A-\mathfrak{P})\right]=\mathfrak{B}+\frac{1}{2} \Sigma\left(a^{2} A\right)-\frac{1}{2} \Sigma\left(a^{2} \mathfrak{R}\right)=\mathfrak{W}-\mathfrak{I}$ 102. $W=\mathfrak{B}+\frac{1}{2} \Sigma\left[a_{1}^{2}(A-\mathfrak{A})\right]+\frac{1}{2} \mathfrak{b}^{2}(2 \pi-\mathfrak{q})$,

103. $w=\mathfrak{B}+\frac{1}{2} \Sigma\left[a_{1}^{2}(A-\mathfrak{A})\right]$,

104. $W=w+\frac{1}{2} \mathfrak{s}^{2}(2 \pi-\mathfrak{q})$,

105. $\left\{\begin{aligned} \mathfrak{W} & =\mathfrak{w}+\pi s^{2}, \\ \mathfrak{T} & =\mathrm{t}+\frac{1}{2} \mathfrak{q} s_{1}^{z},\end{aligned}\right.$

106. $W=\mathfrak{w}+\pi s^{2}-t-\frac{1}{4} \mathfrak{q} s_{1}^{2}$,

wobei $w$ und die Strahlen $a_{1}$ (d. i. $a_{1}, b_{1}, c_{1}, \ldots$. ) sich auf den Punct $\mathbb{S}_{\text {, }}$ dagegen $w$ und $s, \mathrm{t}$ und $s_{1}$ auf die Puncte $S, S_{1}$ beziehen, und die drei Puncte $S, S_{1}$ und $\mathcal{S}$ die Schwerpuncte der Ecken $\boldsymbol{A}, \boldsymbol{B}, \boldsymbol{C}, \ldots$. des Vielecks $\mathfrak{B}$ sind, wenn ihnen beziehlich die Winkel 1) $A, B, C, \ldots$, 2) $\mathfrak{A}, \mathfrak{B}, \mathfrak{C}, \ldots$ und 3) $\boldsymbol{A}-\mathfrak{A}, \boldsymbol{B}-\mathfrak{B}, \boldsymbol{C}-\mathfrak{E}, \ldots$. als Coefficienten zugehören. - Der gegenwärlige Schwerpunct $\mathfrak{S}$ ist hiernach von dem obigen gleichnamigen ( $\mathbb{S}$ XXX.) im Allgemeinen wesentlich verschieden. Aus den vorstehenden Gleichungen folgen übrigens analoge Sätze wie dort. 
8. Steiner, von dem Krümmungs-Schwerpuncte ebener Curven.

\section{§. XXXIII.}

Lärst man die der bisherigen Betrachtung zu Grunde liegenden Vielecke $\mathfrak{B}$ und $\mathfrak{U}$ in Curven $\mathfrak{V}$ and $\mathfrak{U}$ übergehen, wobei jedoch vorläufig $\mathfrak{B}$ geschlossen und stetig convex, dagegen $\mathfrak{U}$ nur längs des Bogens $\mathfrak{A \mathfrak { A }}_{\boldsymbol{1}}$ (Fig. 10.), so weit jene auf ihr rollt, stetig convex sein soll : so bleiben die obigen Gleichungen offenbar auch noch für den gegenwärtigen Fall gültig, so dals man also auch für diese Curven unmittelbar hat (\$. XXX. und XXXI.) :

$$
\begin{aligned}
& \text { 107. } W=\mathfrak{B}+\frac{1}{2} \Sigma\left[a^{2}(A+\mathfrak{A})\right]=\mathfrak{W}+\mathfrak{T}, \\
& \text { 108. } W=\mathfrak{W}+\frac{1}{2} \Sigma\left[a_{1}^{2}(A+\mathfrak{A})\right]+\frac{1}{2} \mathfrak{s}^{2}(2 \pi+\mathfrak{q}), \\
& \text { 109. } w=\mathfrak{W}+\frac{1}{2} \Sigma\left[a_{1}^{2}(A+\mathfrak{A})\right], \\
& \text { 110. } W=w+\frac{1}{2} \mathfrak{s}^{2}(2 \pi+\mathfrak{q}), \\
& \text { 111. }\left\{\mathfrak{W}=\mathfrak{w}+\pi s^{2}\right. \\
& \text { 112. } W=\mathfrak{W}=\mathfrak{w}+\pi s^{2}+\mathfrak{t} \mathfrak{q} s_{1}^{\mathfrak{z}}
\end{aligned}
$$

Der Weg jedes mit der Curve $\mathfrak{Y}$ verbundenen Punctes $\boldsymbol{P}$ - der früher aus einer Reihe Kreisbogen bestand - wird hier irgend eine Curve $\boldsymbol{P P _ { 1 }}$, so dafs die von $\boldsymbol{P}$ beschriebene Figur $\boldsymbol{W}$ von zwei gleichen Geraden $\boldsymbol{P} \mathfrak{A}, \boldsymbol{P}_{1} \mathfrak{A}_{1}$ und zwei Curven $\boldsymbol{P P}_{1}, \mathfrak{A P}_{1}$ begrenzt wird, wovon die letztere, als Basis, allen Figuren $W$ gemein und gleich dem Umfange der Curve $\mathfrak{B}$ ist.

Der eigenthümliche Punct $\mathfrak{S}$, weIchem die Figur $w$ vom kleinsten Inhalte entspricht, behält seine frühere Eigenschaft: nämlich er ist der Schwerpunct der Curve $\mathfrak{B}$, wenn ihren einzelnen Puncten Coefficienten zugeordnet sind, die sich verhalten wie die Summen der unendlich kieinen Winkel, welche die Curven $\mathfrak{Y}$ und $\mathfrak{U}$ in den correspondirenden Puncten mit der Tangente bilden, oder wie die Summen der correspondirenden Krümmungen beider Curven (vergl. \$. XXVIII. u. XXX.). Oder nach (\$. XXXI.) kann der Punct $\mathfrak{S}$ wie folgt gefunden werden. Nämlich von den zwei Puncten $\boldsymbol{S}$ und $\boldsymbol{S}_{1}$, welche dort zu Hülfe genommen worden, ist hier der erste, $\mathbb{S}$, der Krümmungs-Schwerpunct der Curve. $\mathfrak{B}$ ( $\$$. XXII.); der andere, $\boldsymbol{S}_{\boldsymbol{1}}$, ist Schwerpunct derselben, wenn ihren einzelnen Puncten Coefficienten gegeben werden, die sich verkehrt verhạlten wie die Krümmungsradien der Basis $\mathfrak{U}$ in den correspondirender Puncten. Der Punct $\mathfrak{S}$ ist alsdaun der Schwerpunct der Puncte $\boldsymbol{S}$ und $\boldsymbol{S}_{1}$, insofern diesen be- 
8. Steiner, von dem Krïmmungs-Schwerpuncte sbener Curven.

ziehlich die Coefficienten $2 \pi$ und $\mathfrak{q}$ zugeordnet werden, so dafs also $\subseteq$, wie früher, durch die Gleichung :

$$
S \mathfrak{S}: S_{1} \mathfrak{S}=q: 2 \pi
$$

gefunden wird, wo jetzt $\mathfrak{q}$ der Winkel ist, unter welchem die Normalen $\mathfrak{A}$., $\mathfrak{A}_{1} \mathfrak{S}$, der Basis $\mathfrak{U}$, in den Endpuncten des von $\mathfrak{B}$ überrollten Bogens sich schneiden (\$. XXX.).

Die Figur $\mathfrak{W}$ ist die nämliche, welehe bereits in (\$. XXVIII.) näher beschrieben worden. Die Figur $\mathfrak{T}$ entsteht zufolge (S. XXXI.) dadurch, dafs der veränderliche Strahl $P A=a$ (d. h. jede Gerade aus dem festen Pole $P$ nach irgend einem Puncte $A$ der Curve $\mathfrak{B}$ ) auf der Tangente $\mathfrak{A P}$ im correspondirenden Puncte $\mathfrak{A}$ der Basis $\mathfrak{U}$ nach constanter Richtung abgetragen, also $\mathfrak{A P}=a$ genommen wird; wo dann dieses begrenzte Stück der Tangente die Fläche der Figur $\mathfrak{z}=\mathfrak{P P}_{1} \mathfrak{A}_{1} \mathfrak{A P}$ beschreibt, welche somit von zwei Geraden $\mathfrak{X P}, \mathfrak{A}_{1} \mathfrak{P}_{\mathfrak{l}}$ und zwei Curven $\mathfrak{A} \mathfrak{A}_{1}$, $\mathfrak{P} \mathfrak{P}_{1}$ begrenzt wird, von welchen die letztere der Ort des Endpuncts der Tangente ist. Durch $\mathfrak{w}$ und $\mathfrak{t}$ sind die kleinsten Inhalte der Figuren $\mathfrak{W}$ und $\mathfrak{T}$ bezeichnet, die statt finden, wenn diese beziehlich den Schwerpuncten $\boldsymbol{S}$ und $S_{1}$ entsprechen. Endlich sind $s$ und $s_{1}$ die Entfernungen des Punctes $P$ von den Schwerpuncten $\boldsymbol{S}$ und $\boldsymbol{S}_{1}$.

Die obigen Gleichungen enthalten hiernach, unter andern, folgenden Satz:

„Wenn in einer Ebene eine geschlossene, stetig convexe Curve $\mathfrak{B}$ auf einer beliebigen festen convexen Curve $\mathfrak{U}$ rollt, bis sie vieder mit dem anfünglichen Puncte (A) auf dieser aufliegt, so beschreibt jeder mit ihr verbundene $P$ unct $\boldsymbol{P}$ irgend eine Figur $\boldsymbol{W}$, deren Inhalt dann ein Minimum $=w$ wird, wenn der beschreibende Punct der oben genannte Schwerpunct $\mathfrak{S}$ der Curve $\mathfrak{B}$ ist. Puncte $\boldsymbol{P}$, welche von diesem Schwerpuncte $\mathcal{S}$ gleich weit abstehen, beschreiben Figuren $W$ von gleichem Inhalte, und auch umgekehrt; und zwar übertrifft dieser Inhalt jenes Minimum $w$ jedesmal gerade um den Kreissector, welcher den Abstand $\mathfrak{s}$ des Punets $\boldsymbol{P}$ von $\mathfrak{S}$ zum Radius und den constanten Winkel $2 \pi+\mathfrak{q}$ zum Centriwinkel hat (110)."

Ueber die Figur $\mathfrak{S}$ wird im Folgenden ein allgemeiner Satz aufgestellt werden.

Anmerkung. Rollt die Curve $\mathfrak{B}$ auf der concaven Seite der Basis $\mathfrak{U}$, und findet dabei der besondere Umstand Statt, dafs in je zwei 
entsprechenden Puncten beider Curven, die erste $\mathfrak{B}$ gröfsere Krümmung hat, als die andere $\mathfrak{U}$, so erhält man analoge Gleichungen, wie vorhin, nämlich man hat nur in diesen $-\mathfrak{A},-\mathfrak{q},-\mathfrak{T},-\mathfrak{t}$ beziehlich statt $+\mathfrak{A}$, $+\mathfrak{q},+\mathfrak{T},+\mathfrak{t}$ zu setzen, um jene zu erhalten (\$. XXXII.). Auch wenn umgekehrt die Curve $\mathfrak{B}$ in jedem Puncte kleinere Krüımung hat, als die Basis $\mathfrak{U}$ im entsprechenden Puncte, lassen sich analoge Formeln aufstellen.

\section{ङ. XXXIV.}

Die vorstehende Betrachtung (\$. XXXIII.) kann dadurch verallgemeinert werden, dafs man die Bedingung: „die Curve $\mathfrak{B}$ solle geschlossen sein und so lange rollen, bis sie wieder mit dem anfänglichen Puncte auf der Basis $\mathfrak{U}$ aufliege" wegläfst und vielmehr annimmt, sie rolle um einen beliebigen Bogen, etwa um den Bogen $\boldsymbol{A C B}=\mathfrak{A}(\mathfrak{S} \mathfrak{B}$ (Fig. 11.), dabei jedoch immer noch die Bedingung festhält: „dafs von den beiden Bogen, dem rollenden $\boldsymbol{A B}$ und dem überrollten festen $\mathfrak{P} \mathfrak{B}$, keiner einen singulären Punct habe." Unter diesen Umständen gelangt man in der That zu umfassenderen Resultaten und es sind dieselben durch das nämliche einfache und anschauliche Verfahren zu beweisen, wie die bisherigen.

Denn eben so, wie vorhin, folgt auch für den gegenwärtigen Fall, dals die von irgend einem mit der rollenden Curve $\boldsymbol{A B}$ (oder $\mathfrak{B}$ ) verbundenen Puncte $\boldsymbol{P}$ beschriebene Figur $\boldsymbol{W}=\boldsymbol{P P}_{1} \mathfrak{B} \mathfrak{S} \boldsymbol{P}$, ihrem Inhalte nach, gleich ist der Summe zweier anderen Figuren $\mathfrak{W}=\boldsymbol{P} \boldsymbol{A P P} \mathfrak{B P}_{2}$ und $\mathfrak{T}=\mathfrak{A P P}_{\mathfrak{1}} \mathfrak{B} \mathfrak{S} \mathfrak{A}$, welche auf die früher angegebene Weise entstehen (\$. XXVIII. und \$.XXXIII.). Die Figur $\mathfrak{B}$ besteht aber selbst aus zwei anderen Figuren $\boldsymbol{F}$ und $\boldsymbol{T}$, von welchen die erste $\boldsymbol{F}=$ Sector $\boldsymbol{P A C B P}$, und die andere $T=A \mathfrak{P P}_{2} B C A$, so dafs also

$$
\text { 113. } \boldsymbol{W}=\boldsymbol{F}+\boldsymbol{T}+\mathfrak{T} \text {. }
$$

Für die Figuren $\boldsymbol{T}$ und $\mathfrak{s}$, jede für sich betrachtet, hat man zunächst, gemäls dem Früheren, nachstehende Formeln :

$$
\begin{aligned}
& \text { 114. } T=\frac{1}{2} \Sigma\left(a^{2} \boldsymbol{A}\right)=\frac{1}{2} \Sigma\left(a_{1}^{2} \boldsymbol{A}\right)+\frac{1}{2} q s^{2}, \\
& \text { 115. } \boldsymbol{T}=\frac{1}{2} \Sigma\left(a^{2} \mathfrak{A}\right)=\frac{1}{2} \Sigma\left(a_{1}^{2} \mathfrak{A}\right)+\frac{1}{2} \mathfrak{q} s_{1}^{2}, \\
& \text { 116. } t=\frac{1}{2} \Sigma\left(a_{1}^{2} \boldsymbol{A}\right), \text { und } t=\frac{1}{2} \Sigma\left(a_{1}^{2} \mathfrak{A}\right), \\
& \text { 117. } T=t+\frac{1}{2} q s^{2}, \text { und } \boldsymbol{T}=\mathrm{t}+\frac{1}{2} \mathfrak{q} s_{1}^{2},
\end{aligned}
$$

wobei $t$ und $t$ die kleinsten Werthe von $T$ und $\mathfrak{T}$ bezeichnen, welche Statt finden, wenn der Pol $\boldsymbol{P}$ beziehlich mit dem Schwerpuncte $\boldsymbol{S}$ oder $\boldsymbol{S}_{\mathbf{1}}$ zusammenfällt, d.h. mit dem Krümmungs-Schwerpuncte $\boldsymbol{S}$ des Bogens $\boldsymbol{A B}$, 
oder mit dem Schwerpuncte $\boldsymbol{S}_{\mathbf{i}}$ desselben Bogens, wofern die Gewichte seiner einzelnen Puncte sich verhalten, wie die Krümmungen des Bogens $\mathfrak{A P}$ in den correspondirenden Puncten. Der Strahl $a_{1}$ repräsentirt die Abstände sowohl des Punctes $\boldsymbol{S}$ als des Punctes $\boldsymbol{S}_{1}$ von den verschiedenen Puncten des Bogens $\boldsymbol{A B} ; \boldsymbol{s}$ und $s_{1}$ sind die Entfernungen des Punctes $\boldsymbol{P}$ von $\boldsymbol{S}$ und $S_{1}$; und endlich sind $q$ und $q$ die Winkel zwischen den Normalen $A Q$ und $\boldsymbol{B Q}, \mathfrak{A}$, und $\mathfrak{B}$, in den Endpuncten der Bogen $\boldsymbol{A B}, \mathfrak{A} \mathfrak{B}$.

In der Geraden $\boldsymbol{S} \boldsymbol{S}_{1}=d$ nehme man den Punct $\mathfrak{S}$ so, dafs

$$
\boldsymbol{S} \mathfrak{S}: \mathbb{S}_{1} \boldsymbol{S}=\mathfrak{q}: q \text {, }
$$

dafs also $\mathfrak{S}$ der Schwerpunct vion $\boldsymbol{S}$ und $\boldsymbol{S}_{1}$ ist, wenn diesen die Coefficienten $q$ und $q$ zugehören, (oder der Schwerpunct des Bogens $\boldsymbol{A B}$ in Rücksicht der Krümmungs-Summen beider Bogen $\boldsymbol{A B}$ und $\mathscr{A} \mathfrak{B}$ in ihren entsprechenden Puncten). Wird ferner $P \widetilde{S}=\mathfrak{s}$ gesetzt, so hat man für die Summe beider Figuren $\boldsymbol{T}$ und $\mathfrak{T}$ :

118. $T+\mathfrak{T}=t+\mathrm{t}+\frac{1}{2} q s^{2}+\frac{1}{2} \mathfrak{q} s_{1}^{2}=t+\mathrm{t}+\frac{1}{2} \frac{q \mathfrak{q}}{q+q} d^{2}+\frac{1}{2}(q+q) \dot{s}^{2}$,

119. $T_{1}+\mathfrak{T}_{2}=t+t+\frac{1}{2} \frac{q q}{q+q} d^{2}$,

120. $T+\mathfrak{T}=T_{1}+\mathfrak{T}_{1}+\frac{1}{2}(q+q) \dot{\mathfrak{s}}^{2}$, wo $\boldsymbol{T}_{1}$ und $\mathfrak{T}_{1}$ die Stelle von $\boldsymbol{T}$ und $\mathfrak{T}$ in dem Falle vertreten, wenn $\boldsymbol{P}$ in den genannten Schwerpunct $\mathfrak{S}$ fällt, und in welchem Falle, wie man sieht, die Summe $\boldsymbol{T}+\mathfrak{T}$ ein Minimum wird (120).

Nun kann ferner der Sector $\boldsymbol{F}$ immer als Differenz (oder als Summe) zweier andern Figuren angesehen werden, nämlich des Segments $\boldsymbol{A C B D A}$ $=\boldsymbol{G}$ und des Dreiecks $\boldsymbol{A P B}=\frac{1}{2} b y$, dessen gegebene Grundlinie $\boldsymbol{A B}=\boldsymbol{b}$ und die veränderliche Höhe $P E=y$; so dafs also

$$
\boldsymbol{F}=\boldsymbol{G}-\frac{1}{2} b y \text {. }
$$

Hierdurch und vermöge (120) geht die Formel (113) in folgende über :

121. $W=G+T_{1}+\mathfrak{T}_{1}+\frac{1}{2}(q+q) \mathfrak{s}^{2}-\frac{1}{2} b y$,

wo rechts alle Gröfsen, aufser $\mathfrak{s}$ und $y$, constant sind. Diese zwei Veränderlichen lassen sich aber durch eine einzige ersetzen. Aus $\mathbb{S}$ auf die Sehne $\boldsymbol{A B}$ fälle man das Perpendikel $\mathfrak{S} D=p$, nehme in der Verlängerung desselben, hinter $\mathfrak{S}$, den Punct $R$ so, dafs

$$
\text { 122. } \quad \boldsymbol{S R}=\frac{b}{2(q+q)} \text {, }
$$

so ist, wenn $P R=r$ gesetzt wird - (durch Hülfe des Perpendikels von $P$ auf $(S D): r^{2}-\mathfrak{b}^{2}=(R D-y)^{2}-(S D-y)^{2}$, und daraus folgt: 
123. $\frac{1}{2}(q+q) b^{2}-\frac{1}{2} b y=\frac{1}{2}(q+q) r^{2}-\frac{1}{4} b\left(2 p+\frac{b}{2(q+q)}\right)$, und mithin (121):

124. $W=G+T_{1}+\mathfrak{T}_{1}-\frac{1}{4} b\left(2 p+\frac{b}{2(q+q)}\right)+\frac{1}{2}(q+q) r^{2}$,

wo nunmehr rechts $r$ die einzige veränderliche Grölse ist. Der Inhalt der Figur $\boldsymbol{W}$ ändert sich demnach mit der Entfernung $\boldsymbol{r}$ des beschreibenden Punctes $\boldsymbol{P}$ von dem ausgezeichneten Puncte $\boldsymbol{R}$ zugleich, und zwar ist seine $Z$ u - oder Abnahme dem Quadrate dieser Entfernung proportional, so dafs $W$ ein Minimun wird, $=w$, wenn $r=0$, d. h. wenn $\boldsymbol{P}$ in $\boldsymbol{R}$ fällt. Also ist:

125. $w=G+T_{1}+\mathfrak{T}_{1}-\frac{1}{4} b\left(2 p+\frac{b}{2(q+q)}\right)$, und 186. $W=w+\frac{1}{2}(q+q) r^{2}$.

Die wesentlichsten Sätze aus dieser Betrachtung sind folgende:

a. ,Wenn in einer Ebene ein beliebiger, stetig convexer Curvenbogen $\boldsymbol{A B}$ auf der convexen Seite irgend eines anderen stetig convexen festen Curvenbogens $\mathfrak{A B}$ rollt, so beschreibt jeder mit der rollenden Curve fest verbundene Punct $\boldsymbol{P}$ irgend eine Figur $\boldsymbol{W}$, deren Inhalt dann ein Minimum, $=w$, wird, wenn jener Punct der oben construirte besondere $\boldsymbol{P}$ unct $\boldsymbol{R}$ ist. Puncte $\boldsymbol{P}$, welche gleich weit von diesem eigenthümlichen $\boldsymbol{P}$ uncte $\boldsymbol{R}$ entfernt sind, also in irgend einer um $\boldsymbol{R}$ beschriebenen Kreislinie liegen, erseugen gleich gro/se Figuren $W$, und auch umgekehrt; und zwar ist ihr Inhalt gerade um den Sector des genannten Kreises, dessen Centriwinkel $=q+\mathfrak{q}$, also constant ist, grö/ser als jener kleinste Inhalt $w$ (126)."

b. 1) ,Bewegt sich eine veränderliche Tangente $A \mathfrak{P}$ an einem stetig convexen Curvenbogen $A C B$ unter der Bedingung, da/s sie in jedem Augenblicke dem Strahle $\boldsymbol{P} \boldsymbol{A}$ gleich ist, welcher ihren Berührungspunct $(\boldsymbol{A})$ mit irgend einem festen Pole $\boldsymbol{P}$ in der Ebene der Curve verbindet, so beschreibt sie irgend eine Figur $T$, deren Inhalt dann ein Minimum, $=t$, wird, wenn jener Pol der Krümmungs-Schwerpunct $\mathbf{S}$ dos gegebenen Bogens $\boldsymbol{A C B}$ ist. Polen $\boldsymbol{P}$, welche in irgend einer um $\mathbf{S}$ beschriebenen Kreislinie liegen, entsprechen Figuren $\boldsymbol{T}$ von gleichem Inhalte, der jedesmal gerade um einen Sector jenes Kreises, welcher den constanten Winkel q zum Centriwinkel hat, grö/ser ist, als jener kleinste Inhalt $t$ (117)." Und 
2) "Ist aufser dem Bogen $\boldsymbol{A B}$ noch irgend ein anderer stetig convexer Bogen $2(\mathfrak{B}$ von gleicher Länge gegeben, und bewegt sich an demselben die Tangente $2 \mathfrak{P}$ unter der Bedingung, dafs sie stets dem Strahle AP gleich ist, welcher den ihrem Berührungspuncte correspondirenden Punct in der Curve $\boldsymbol{A B}$ mit dem festen Pole $\boldsymbol{P}$ verbindet: so beschreibt sie irgend eine Figur $\mathfrak{T}$, deren Inhalt ein Minimum word, $=t$, wenn der Pol der oben bestimmte Schwerpunct $\mathcal{S}_{\mathbf{1}}$ des Bogens $\boldsymbol{A B}$ ist; liegt der Pol $\boldsymbol{P}$ in irgend einer um $\boldsymbol{S}_{1}$ beschriebenen Kreislinie, so nimmt der Inhalt von $\mathfrak{T}$ gerade um einen Sector dieses Kreises, dessen Centriwinkel dem constanten Winkel q gleich ist, zu (117)."

3) „Werden, für einen und denselben Pol $\boldsymbol{P}$, die beiden Figuren $\boldsymbol{T}$ und $\mathfrak{T}$ zugleich betrachtet, so ist ihre Summe $\boldsymbol{T}+\mathfrak{T}$ dann ein Minimum $=\boldsymbol{T}_{1}+\mathfrak{T}_{1}$, wenn der Pol der Schwerpunct $\mathbb{S}$ ist (d. h. der Schwerpunct des Bogens $\boldsymbol{A B}$ in Rücksicht der Krümmungs-Summen beider Bogen $\boldsymbol{A B}$ und $\mathscr{U} \mathfrak{B}$ in den correspondirenden Puncten, oder der Scliwerpunct der Puncte $S$ und $S_{1}$ in Rücksicht der Coefficienten $q$ und $q$ ). Liegt aber der Pol $\boldsymbol{P}$ in einer Kreislinie, deren Mittelpunct $\mathfrak{G}$, so nimmt die Summe $\boldsymbol{T}+\mathfrak{T}$ um einen Sector dieses Kreises zu, dessen Centriwinkel immer $=q+\mathfrak{q}$ ist (120)."

Anmerkung 1. Die Tangente $A P$ oder $\mathfrak{A P}$ kann, vom Berührungspuncte aus, nach zwei entgegengesetzten Richtungen genommen werden, wodurch zugleich zwei verschiedene Figuren $\boldsymbol{T}$ und $\boldsymbol{T}_{1}$, oder $\mathfrak{T}$ und $\mathfrak{F}_{1}$ entstehen, aber jedesmal haben beide unter sich gleichen Inhalt, so dafs immer $T=T_{1}$, oder $\mathfrak{Z}=\mathfrak{T}_{1}$ (vergl. $\$$. XXVIII.).

8. Der letzte Satz $(b, 3$.$) findet ähnlicherweise statt, wenn aufser$ dem Bogen $\mathfrak{A} \mathfrak{C B}$ noch mehrere andere Bogen $\mathfrak{A}_{1} \mathfrak{B}_{1}, \mathfrak{A}_{2} \mathfrak{B}_{2}, \ldots$ unter denselben Bedingungen gegeben sind, denen dann ebenfalls Schwerpuncte $\boldsymbol{S}_{2}, \boldsymbol{S}_{3}, \ldots$, so wie Winkel $\mathfrak{q}_{1}, \mathfrak{q}_{2}, \ldots$ und Figuren $\mathfrak{T}_{1}, \mathfrak{T}_{2}, \ldots$ entsprechen. Nämlich eben so wird alsdann die Summe $\boldsymbol{T}+\mathfrak{T}+\mathfrak{T}_{1}+\mathfrak{Z}_{2}+\ldots$ ein Minimum $=m$, wenn der Pol $\boldsymbol{P}$ in den Schwerpunct $\mathfrak{S}$ der Puncte $\boldsymbol{S}, \boldsymbol{S}_{1}, \boldsymbol{S}_{2}, \boldsymbol{S}_{3}, \ldots$ fällt, wofern diesen die Coefficienten $q, \mathfrak{q}, \mathfrak{q}_{1}, \mathfrak{q}_{2}, \ldots$ zugeordnet sind; und aufserdem hat man für einen beliebigen $\mathrm{Pol} \boldsymbol{P}$, wenn $\boldsymbol{P} \widetilde{S}=\boldsymbol{r}$ gesetzt wird, die Relation:

127. $\boldsymbol{T}+\mathfrak{i}+\mathfrak{T}_{1}+\mathfrak{T}_{2}+\ldots . .=m+\frac{1}{2}\left(q+\mathfrak{q}+\mathfrak{q}_{1}+\mathfrak{q}_{2}+\ldots ..\right) \boldsymbol{r}^{2}$.

Die Richtigkeit dieser Angaben folgt leicht aus (S. VII.). 
8. Soll in Ansehung des obigen Satzes (a.), unter allen Puncten $\boldsymbol{P}$, die in der rollenden Curve $\mathfrak{B}$ (wovon $\boldsymbol{A C B}$ nur ein begrenztes Stück ist) selbst liegen, derjenige gefunden werden, welcher die kleinste oder grörste Figur $W$ beschreibt, so ist klar, dafs derselbe nur der Fufspunct einer Normale sein kann, die aus dem eigenthümlichen Puncte $\boldsymbol{R}$ auf die Curve $\mathfrak{B}$ gefället wird. Eben so verhält es sich, wenn $\operatorname{der} P$ unct $\boldsymbol{P}$ in irgend einer anderen, in der Ebene der $\mathfrak{B}$ gegebenen Curve liegen soll. - Dasselbe kann auch über die. Sätze $(b$. $)$ bemerkt werden.

\section{S. XXXV.}

Durch die vorstehende Betrachtung (\$. XXXIV.) sind wir zu den allgemeinsten Resultaten gelangt. Denn nicht nur umfassen dieselben die meisten früheren als bescudere Fälle, sondern es folgen daraus noch zahlreiche andere, specielle Sätze, wofern man nämlich in Rücksicht der gegebenen Elemente bestimmte Einschränkungen und Modificationen eintreten lä[st *). Dahin gehört unter andern : dafs die Winkel $q$ und q bestimmte Werthe haben (wie z. B. wenn $y=2 \pi$ und die Curve $\mathfrak{B}$ geschlossen, also die Sehne $\boldsymbol{A B}=0$ ist, wodurch man zu den Resultaten in $\mathfrak{\text { .XXXIII. }}$ gelangt): dafs die eine oder die andere gegebene Curve $\mathfrak{B}$ oder $\mathfrak{U}$ in eine Gerade übergeht: dafs ferner die eine oder die andere, oder dafs beide zugleich in bestimmte einfache Curven übergehen, etwa in Kreise, $u$. s. w.

Von solchen speciellen Sätzen mögen hier noch folgende Platz finden :

\section{Wenn die Basis $\mathfrak{A B}$ eine Gerade wird und}

\section{1. $\mathrm{ACB}$ ein beliebiger Curvenbogen bleibt.}

In diesem Falle wird $\mathfrak{q}=0, \mathfrak{I}=0$ und $\boldsymbol{S}_{1}$ verschwindet oder kommit nicht in Betracht, so dafs $\mathcal{S}$ mit $\boldsymbol{S}$ zusammenfallt. Daher wird der ausgezeichnete Punct $\boldsymbol{R}$ gefunden, wenn man aus dem Krümmungs-Schwerpuncte $S$ des rollenden Bogens $A B$ auf die Sehne $A B$ das Perpendikel $S D$ fället und auf dessen Verlängerung, hinter $S$, den Punct $R$ so nimmt, dafs (122):

$$
\text { 128. } R S=\frac{b}{2 q} \text {. }
$$

*) Da man sich, in älterer und in neuerer Zeit, so vielfach mit Betrachtung der aurch Rollen erzeugten Curven (Roulettes) beschäftigt hat, so dürfte es wohl auffallend scheinen, dafs das obige einfache und allgemeine Gesetz, dem die Quadratur je eines Bystems solcher Curven unterworfen ist, so lange verborgen bleiben konnte. 
Die obige Formel (126) reducirt sich hier auf folgende:

129. $W=w+\frac{1}{2} q r^{2}$.

Das heifst :

„Rollt ein stetig convexer Curvenbogen $\boldsymbol{A B}$ auf einer festen Geraden $\mathfrak{A B}$, so beschreibt jeder mit ihm verbundene Punct $\boldsymbol{P}$ irgend eine Figur $W$, die am kleinsten wird, $=w$, wenn jener Punct der vorgenannte Punct $\boldsymbol{R}$ ist. Puncte $\boldsymbol{P}$, welche in irgend einer um $\boldsymbol{R}$ beschriebenen Kreislinie liegen, erzeugen Figuren $W$, deren Inhalt gerade um einen Sector des Kreises, dessen Centriwinkel $=q$, grö/ser als jener kleinste Inhalt w ist."

Anmerkung. Da auch hier, eben so wie (\$. XXI.), die Figur $W$ allemal gerade doppelt so grofs ist, als die dem nämlichen Puncte $\boldsymbol{P}$ entsprechende Fufspuncten - Figur $\boldsymbol{V}$ in Bezug auf den gegebenen Bogen $\boldsymbol{A B}$, was sich gleicherweise zeigen läfst, so ist die Figur $V$ demselben Gesetze unterworfen, wie die Figur $W$, d. h. „ihr Inhalt wird ein Minimum, $=v$, wenn sie dem ausgezeichneten Puncte $\boldsymbol{R}$ entspricht; für einen beliebigen andern Punct $\boldsymbol{P}$, wenn $\boldsymbol{P R}=\boldsymbol{r}$ gesetzt wird, ist

$$
\text { 130. } V=v+\frac{1}{1} q r^{2} \text {, }
$$

also die Inhalts-Zunahme gerade die Hälfte des Kreissectors, der $r$ zum Radius und q zum Centriwinkel hat."

2. Wenn $\boldsymbol{A B}$ insbesondere ein Kreisbogen ist.

Dann wird $\boldsymbol{Q}$ der Mittelpunct des Kreises, also $\boldsymbol{q}$ der Centriwinkel über dem Bogen $\boldsymbol{A B}$, und dann fällt der Krümmungs-Schwerpunct $\boldsymbol{S}$ offenbar mit dem gewöhnlichen Schwerpuncte des Bogens $\boldsymbol{A B}$ zusammen, so dafs sein Abstand vom Mittelpunct, wie bekannt:

131. $Q S=\frac{b}{q}$.

Diese Gerade $Q S$ steht auf der Sehne $A B=b$ senkrecht; daher liegt auch der ausgezeichnete Punct $R$ in ihr, und seine Entfernung vom Mittelpuncte $Q$ ist (128 u. 131):

$$
\text { 138. } Q R=Q S+S R=\frac{3 b}{2 q},
$$

also : "gleich der dreifachen Sehne dividirt durch den doppelten Centriwinkel." Man erkeunt daraus, dafs $\boldsymbol{R}$ sowohl innerhalb als jenseits des Kreises liegen kann, nachdem nämlich $3 b<2 q a$ oder $3 b>2 q a$, wenn $a$ 
der Radius des Kreises ist. Ist $3 b=2 q a=2 A C B$, also der Bogen gerade anderthalb mal so grofs, als die Sehne, so fällt $\boldsymbol{R}$ in den Bogen $\boldsymbol{A B}$ selbst und zwar in dessen Mitte.

Da $\mathfrak{s}=0$ (1), so ist (113):

$$
\boldsymbol{W}=\boldsymbol{F}+\boldsymbol{T}
$$

und wenn $\boldsymbol{P}$ im Mittelpuncte $\boldsymbol{Q}$ des Kreises liegt, so ist

$$
\boldsymbol{F}=\frac{1}{2} q a^{2}, \quad \text { und (114) } \quad \boldsymbol{T}=\frac{1}{2} q a^{2},
$$

daher ist für diesen besondern Fall (was auch unmittelbar folgt, da die von $\boldsymbol{Q}$ beschriebene Figur $W_{1}$ ein Rechteck ist, dessen Seiten $a$ und $q a$ $=A C B$ sind) :

$$
W_{1}=q a^{2},
$$

und daher folgt für die von $R$ beschriebene kleinste Figur (129 u. 131):

133. $w=q a^{2}-\frac{1}{2} q\left(\frac{3 b}{2 q}\right)^{2}=q a^{2}-\frac{9}{8 q} b^{2}=a^{2}\left(q-\frac{9}{2 q} \sin \frac{1}{2} q^{2}\right)$.

Für die von einem beliebigen Puncte $\boldsymbol{P}$ beschriebene Figur folgt nunmehr (129):

$$
\text { 134. } W=q a^{2}-\frac{9}{8 q} b^{2}+\frac{1}{2} q r^{2} \text {. }
$$

Die Figuren $W$ und $w$ sind hier bestimmte Stücke von gewöhnlichen Cykloiden (gestreckte oder verkürzte), nämlich solche Stücke, welche von einem Cykloidenbogen $\boldsymbol{P P}_{1}$, den beiden Normalen in seinen Endpuncten, $\boldsymbol{P} \mathfrak{A}$ und $\boldsymbol{P}_{\mathfrak{1}} \mathfrak{B}$, und der zwischen den letzteren liegenden (geradlinigen) Strecke $\mathfrak{A} \mathfrak{B}$ der Basis begrenzt werden. Die Formeln (134 und 133) geben die Quadratur dieser Stücke mittelst der gegebenen Elemente.

In dem oben genannten besonderen Falle, wo $3 b=2 q a$ ist und $\boldsymbol{R}$ in die Mitte des Bogens $\boldsymbol{A B}$ fällt, besteht die kleinste Figur $\boldsymbol{w}$ aus zwei einander gleichen Sectoren der sogenannten gemeinen Cykloide, und alsdann ist

$$
W=\frac{1}{2} q\left(a^{2}+r^{2}\right) \text {. }
$$

Insbesondere kann auch $w=0$ werden, nämlich in dem Falle, wo $q a: b=3: \sqrt{ } 8$ (133), d. h. wo der Bogen $A C B$ sich zur Sehne $A B$ verhält, wie $3 \mathrm{zu} r 8$. Alsdann ist $W=\frac{1}{2} q r^{2}$, und $R$ liegt jenseits des Kreises. 
II. Wenn $\mathrm{ACB}$ in eine Gerade übergeht und

1. die Basis $\mathfrak{A B}$ eine beliebige Curve bleibt.

In diesem Falle ist offenbar $T=0, G=0$ und $q=0$, und vermöge des Letzteren verschwindet der Punct $\boldsymbol{S}_{\text {; }}$ daher vereinigt sich der Punct $\mathcal{S}$ mit $S_{1}$, dieser aber liegt in der Geraden $\boldsymbol{A B}$ selbst, nämlich er ist ihr Schwerpunct, wenn sie so schwer gedacht wird, dafs die Gewichte ilırer einzelnen Puncte sich verhalten, wie die Krümmungen der Basis $\mathfrak{A} \mathfrak{B}$ in den correspondirenden Puncten. Daher wird ferner der ausgezeichnete Punct $R$ erhalten, wenn man in dem Puncte $\boldsymbol{S}_{\mathbf{1}}$ auf die Gerade $\boldsymbol{A B}=\boldsymbol{b}$ ein Perpendikel errichtet (nach der Basis $\mathfrak{A}(\mathfrak{B}$ hin), und in demselben $\boldsymbol{R}$ so nimmt, dals (122)

$$
\text { 135. } \quad S_{1} R=\frac{b}{2 \mathfrak{q}}=\beta \text {. }
$$

Hiernach reduciren sich die obigen Formeln (125 u. 126) - da auch $\boldsymbol{p}=0$, weil $\boldsymbol{S}_{1}$ in $\boldsymbol{A B}$ liegt - auf folgende :

$$
\text { 136. } w=\mathrm{t}-\frac{1}{4} b \frac{b}{i q}=\mathrm{t}-\frac{1}{2} \mathfrak{q} \beta^{2} \text {, }
$$

137. $W=w+\frac{1}{2} \mathfrak{q} r^{2}=\mathrm{t}-\frac{1}{8 \mathfrak{q}} b^{2}+\frac{1}{2} \mathfrak{q} r^{2}=\mathrm{t}+\frac{1}{2} \mathfrak{q}\left(r^{2}-\beta^{2}\right)$.

Also: „Wälst sich eine Gerade $\boldsymbol{A B}$ (von dem einen Endpuncte $A$ bis zum andern $B$ ) auf irgend einer festen, stetig convexen Curve $\mathfrak{A} \mathfrak{B}$, so beschreibt unter allen mit ihr fest verbundenen Puncten (d. h. die ihre Lage gegen die Gerade $\boldsymbol{A B}$, während diese sich bewegt, nicht änderu) der besonders bestimmte Punct $\boldsymbol{R}$ die kleinste Figur $w$; die von irgend einem anderen Puncte $\boldsymbol{P}$ beschriebene Figur $\boldsymbol{W}$ ist jedesmal um den Kreissector, dessen Radius $\boldsymbol{r}=\boldsymbol{P R}$ und dessen Centriwinkel $q \mathrm{C}=\mathrm{dem}$ Winkel zwischen den Normalen in den Endpuncten der Basis $\mathfrak{A} \mathfrak{B})$, grö(ser, als jene."

Für den besonderen Fall, wo $\boldsymbol{r}=\beta$ ist und somit der Punct $\boldsymbol{P}$ in der mit dem Radius $\beta=\boldsymbol{R} \boldsymbol{S}_{1}$ um den Punct $\boldsymbol{R}$ beschriebenen Kreislinie liegt, hat man (137):

$$
\text { 138. } W_{1}=t \text {; }
$$

und in der That fält die von dem Puncte $\boldsymbol{S}_{1}$ beschriebene Figur, welcher Punct in der Kreislinie liegt, mit der Figur $t$ zusammen.

Unter allen Puncten, welche in $\operatorname{der}$ Geraden $\boldsymbol{A B}$ selbst liegen, beschreibt $S_{1}$ die kleinste Figur $t ;$ jeder aber beschreibt eine Evolvente der Curve $\mathfrak{A B}$ (oder vielmehr zwei Bogen derselben, nur der Endpunct $\boldsymbol{A}$ 
oder $\boldsymbol{B}$ beschreibt blofs einen Bogen), so dafs also in diesem Falle die Figur $\boldsymbol{W}$ irgend ein bestimmtes Stück der Evolvente ist (im Allgemeinen zwei Sectoren derselben); zudem fällt $\boldsymbol{W}$ mit der durch $\mathfrak{i}$ bezeichneten Figur zusammen (\$. XXXIV.), und in der That geben die Formeln (117) und (137) für beide den nämlichen Inhalt, indem $r, \beta$ und $s_{1}$ die Seiten eines rechtwinkligen Dreiecks sind und daher $r^{2}-\beta^{2}=s_{1}^{2}$ ist.

2. Wenn die Basis $\mathfrak{A} \mathfrak{B}$ insbesondere ein Kreisbogen ist, dann liegt $\boldsymbol{S}_{1}$ nothwendig in der Mitte der Geraden $\boldsymbol{A B}$. Der Radius der Basis sei $=\alpha$; so ist der überrollte Bogen $\mathfrak{A P}=\mathfrak{q} \alpha=b$, und folglich (135) :

$$
\text { 139. } \beta=\frac{1}{2} \alpha \text {, }
$$

d. h. „der Abstand $\beta$ des ausgezeichneten Punctes $\boldsymbol{R}$ von der Geraden $\boldsymbol{A B}$ ist halb so gro/s, als der Radius a der Basis, so da/s er also constunt bleibt, wenn der letztere gegeben ist, mag die rollende Gerade AB grö/ser oder kleiner angenommen werden; zudem liegt $\boldsymbol{R}$ nach der Basis $\mathfrak{A} 3$ hin und das aus ihm auf $\boldsymbol{A B}$ gefüllte Perpendikel trifft die Mitte $\boldsymbol{S}_{1}$ der letztern."

Die von dem Puncte $\boldsymbol{S}_{1}$ beschriebene Figur t (137) besteht hier aus zwei gleichen Sectoren der Evolvente des Grundkreises, wenn dieser von der Mitte $\mathfrak{S}_{\mathfrak{1}}$ des gegebenen Bogens $\mathfrak{\Re} \mathfrak{B}$ bis zu dessen Endpuncten $\mathfrak{A}$ und $\mathfrak{B}$ abgewickelt wird. Daher ist:

140. $t=\frac{1}{24} \mathfrak{q} b^{2}=\frac{1}{24} \mathfrak{q}(\mathfrak{q} \alpha)^{2}=\frac{1}{6} q^{3} \beta^{2}$, und $(136,137)$ :

141. $w=\frac{\mathfrak{q}^{2}-3}{24 q} b^{2}=\frac{q^{2}-3}{24} q \alpha^{2}=\frac{q^{2}-3}{6} q \beta^{2}$,

142. $W=\frac{q^{2}-3}{24 q} b^{2}+\frac{1}{2} \mathfrak{q} r^{2}=\frac{q^{2}-3}{24} q a^{2}+\frac{1}{2} \mathfrak{q} r^{2}=\frac{q^{2}-3}{6} \mathfrak{q} \beta^{2}+\frac{1}{2} \mathfrak{q} r^{2}$.

Die von dem Puncte $\boldsymbol{R}$ beschriebene kleinste Figur $\boldsymbol{w}$ kann, wie man sieht (141), negativ oder positiv werden; auch wird insbesondere $w=0$, wenn der Winkel $\mathfrak{q}=\sqrt{3}$, oder $b=a \sqrt{ } 3$; alsdann ist die vou irgend einem Puncte $\boldsymbol{P}$ beschriebene Figur

$$
\text { 143. } W=\frac{1}{2} r^{2} \sqrt{3},
$$

d. h. gleich dem doppelten Inhalte des gleichseitigen Dreiecks über desn Abstande des Punctes $\boldsymbol{P}$ von $\boldsymbol{R}$."

Liegt der Punct $\boldsymbol{P}$ in der rollenden Geraden $\boldsymbol{A B}$ selbst und wird $P S_{1}=s_{1}$ gesetzt, so ist $r^{2}=\beta^{2}+s_{1}^{2}$, und daher hat man (142):

144. $W=\frac{1}{24} q b^{2}+\frac{1}{2} q s_{1}^{2}=\frac{1}{24} q^{3} \alpha^{2}+\frac{1}{2} q s_{1}^{2}=\frac{1}{6} q^{3} \beta^{2}+\frac{1}{2} q s_{1}^{2}$,

Crelle': Journal d. M. Bd. XXI. Hft. 2. 
wo jeizt $W$ ein bestimmtes Stück irgend einer Evolvente des Grundkreises ist, welches von einem Bogen $\boldsymbol{P P}_{1}$ derselben, den Normalen $\boldsymbol{P} \mathfrak{A}_{\mathfrak{l}}$ und $\boldsymbol{P}_{1} \mathfrak{B}$ in dessen Endpuncten, und dem correspondirenden Bogen $\mathfrak{A} \mathfrak{B}$ der Basis begrenzt wird.

Es ist klar, dafs auch in andern Fällen der Schwerpunct $\boldsymbol{S}_{1}$ in die Mitte der Geraden $\boldsymbol{A B}$ fallen kann, wie z. B. wenn die Basis $\mathfrak{A B}$ in Bezug auf eine Axe senkrecht symmetrisch ist, also etwa der Bogen eines Kegelschnitts, in dessen Mitte der Scheitel einer Axe desselben liegt. Von solchen Beispielen mag hier noch das folgende in Betracht kommen, wo nämlich:

3. die Basis $\mathfrak{A} \mathfrak{B}$ ein ganzer Bogen der gemeinen Cykloide ist.

In diesem Falle wird $\mathfrak{q}=\pi$, also $\beta=\frac{b}{2}$, wodurch die Lage des Punctes $\boldsymbol{R}$ (in Rücksicht der rollenden Geraden $\boldsymbol{A B}$ ) vollkommen bekannt ist, indem $\boldsymbol{S}_{\mathbf{1}}$ in der Mitte von $\boldsymbol{A B}$ liegt. Der Radius des Kreises, durch welchen die Cykloide $\mathfrak{H} \mathfrak{B}$ erzeugt worden, sei $\alpha$, so ist bekanntlich $8 \alpha=\mathfrak{A} \mathfrak{B}=$ $\boldsymbol{A B}=\boldsymbol{b}=2 \pi \beta$. Aus einer andern, allgemein bekannten, Eigenschaft der Cykloide folgt leicht, dafs der Inhalt der von $\boldsymbol{S}_{1}$ beschriebenen Figur:

$$
\text { 145. } t=4 \pi \alpha^{2}=\frac{1}{16} \pi b^{2}=\frac{1}{4} \pi^{3} \beta^{2} \text {. }
$$

Daraus folgt weiter (136 und 137):

146. $w=\frac{\pi^{2}-2}{16 \pi} b^{2}=4 \frac{\pi^{2}-2}{\pi} \alpha^{2}=\frac{\pi^{2}-2}{4} \pi \beta^{2}$,

147. $W=\frac{\pi^{2}-2}{16 \pi} b^{2}+\frac{1}{2} \pi r^{2}=4 \frac{\pi^{2}-2}{\pi} \alpha^{2}+\frac{1}{2} \pi r^{2}=\frac{1}{4}\left(\pi^{2}-2\right) \pi \beta^{2}+\frac{1}{2} \pi r^{2}$.

Für die von dem Endpuncte $\boldsymbol{A}$ oder $\boldsymbol{B}$ beschriebene Figur (die Evolvente der Cykloide $\mathfrak{X} \mathfrak{B})$ - für welche $r^{2}=\beta^{2}+\left(\frac{1}{2} b\right)^{2}=\frac{\pi^{2}+1}{4 \pi^{2}} b^{2}-$ hat man:

$$
\text { 148. } W=\frac{3}{16} \cdot \pi b^{2}=12 . \pi \alpha^{2}=\frac{3}{4} \cdot \pi^{3} \beta^{2} \text {. }
$$

III. Wenn $\mathrm{ACB}$ ein Kreisbogen und

1. die Basis $\mathfrak{A} \mathfrak{B}$ eine beliebige Curve ist.

Hier fällt $\boldsymbol{S}$ in den gewöhnlichen Schwerpunct des Bogens $\boldsymbol{A B}$; die übrigen wesentlichen Puncte $\boldsymbol{S}_{1}$, $\mathfrak{S}$ und $R$ werden nicht näher bestimmt; allein ohne dieselben genauer zu kennen, kann doch der Inhalt der dem Mittelpuncte $\boldsymbol{Q}$ des Kreises $\boldsymbol{A B}$ entsprechenden Figuren $\boldsymbol{W}$ und $\mathfrak{T}$ gefunden werden. Denn da für diesen Fall in den obigen Formeln (114 und 115) der Strahl a constant, nämlich gleich dem Radius des Kreises $\boldsymbol{A B}$, so ist : 
8. Steiner, von dem Krümmungs-Schwerpuncte ebener Curven.

$$
\begin{array}{r}
\boldsymbol{T}=\frac{1}{2} \Sigma\left(a^{2} \boldsymbol{A}\right)=\frac{1}{2} a^{2} \Sigma(\boldsymbol{A})=\frac{1}{2} q a^{2}, \text { und } \\
149 . \quad \mathfrak{T}=\frac{1}{2} q a^{2},
\end{array}
$$

und ferner ist der Sector

so dafs folglich (113):

$$
\boldsymbol{F}=\frac{1}{2} q a^{2},
$$

150. $\boldsymbol{W}=\frac{1}{2}(2 q+\mathfrak{q}) \boldsymbol{a}^{2}$.

Hiernach hat man folgende zwei Sätze :

a) ,Bewegt sich eine constante Tangente $\mathfrak{A P}=a$ längs einer festen, stetig convexen Curve $\mathfrak{A} \mathfrak{B}$, so beschreibt sie eine Fiyur $\mathfrak{\mathfrak { I }}$, deren Inhalt einem Kreissector gleich ist, $=\frac{1}{2} q a^{2}(149)$, welcher die Tangente zum Radius und den Winkel zwischen den Normalen in den Endpuncten der Curve zum Centriwinkel hat." - Hierdurch lassen sich verschiedene sogenannte ,Zuglinien" (Tractorien) unmittelbar quadriren.

B) ,Rollt ein Kreis auf der convexen Seite einer festen Curve $\mathfrak{P} \mathfrak{Z}$ (um einen beliebigen Bogen $\boldsymbol{A B}=\mathfrak{A B}$, der kleiner oder gröfser als der Kreisumfang sein kann), so beschreibt sein Mittelpunct $Q$ eine Figur $W$ die allemal dem Sector des Kreises gleich ist, welcher den doppelten Centriwinkel über dem abgerollten Bogen $A B$ und den Winkel zwischen den Normalen in den Endpuncten der Basis $\mathfrak{A B}$ zusammengenommen, zum Centriwinkel hat (150)." - Die vom Mittelpuncte $Q$ des Kreises beschriebene Curve $\boldsymbol{Q} \boldsymbol{Q}_{1}$ und die Basis $\mathfrak{A B}$ heifsen ,parallele Curven." Die Figur $W$ ist ein Stück des Ringes zwischen denselben, begrenzt durch die gemeinschaftlichen Normalen $Q \mathfrak{A}$ und $\boldsymbol{Q}_{1} \mathfrak{B}$. Die Länge der Curve $\boldsymbol{Q} \boldsymbol{Q}_{\mathbf{1}}$ ist $=(q+q) a$, was aus einer andern geometrischen Betrachtung leicht folgt. (Vergl. Abh. von Crelle in Annal. de Math. par Gergonne, tom. XII.)

2. Wenn die Basis $\mathfrak{P} \mathfrak{B}$ auch ein Kreisbogen ist, dann fällt auch $\boldsymbol{S}_{1}$ in den gewöhnlichen Schwerpunct des Bogens $\boldsymbol{A B}$, so dals folglich die drei Puncte $\mathbb{S}, S_{1}$ und $\mathcal{S}$ in demselben vereinigt sind. Nun liegt der eigenthümliche Punct $\boldsymbol{R}$ in dem durch $\mathfrak{S}$ gehenden Durchmesser des Kreises $\boldsymbol{A B}$, und sein Abstand yom Mittelpuncte $\boldsymbol{P}$ des letzteren ist (131 u. 128):

151. $\boldsymbol{Q R}=\frac{b}{q}+\frac{b}{2(q+q)}=\frac{3 q+2 q}{2 q+2 q} \cdot \frac{b}{q}=\frac{2 a+3 \mathfrak{a}}{2 a+2 \mathfrak{a}} \cdot \frac{b}{q}=\frac{3+2 n}{2(1+n)} \cdot \frac{b}{q}=r_{1}$, wo $\mathfrak{a}$ der Radius der Basis $\mathfrak{A} \mathfrak{B}$ und das Verhältnifs der Radien $a: \mathfrak{a}=n$ geselzt ist, (es ist dann auch $q: q=n$ ).

Da hierdurch der Abstand $\left(r_{1}\right)$ des Mittelpuncts $Q$ von dem Puncte $\boldsymbol{R}$ gegeben ist, und da man auch den Inhalt der von ihm beschriebenen Fi- 
gur W kennt (150) : so wird dadurch der Inhalt der von $R$ beschriebenen kleinsten Figur $w$ gefunden, nämlich (126 u. 150):

152. $w=\frac{1}{2}(2 q+q) a^{2}-\frac{1}{2}(q+q) r_{1}^{2}=\frac{1}{2}(2 q+q) a^{2}-\frac{1}{8} \frac{(3 q+2 q)^{2}}{q+q} \cdot\left(\frac{b}{q}\right)^{2}$

$$
\begin{aligned}
& =\frac{1}{2} q a^{2}+\frac{1}{2}(q+q)\left(a^{2}-r_{2}^{2}\right)=\frac{1}{2} \frac{a+2 a}{\mathfrak{a}} q a^{2}-\frac{1}{8} \frac{(2 a+3 a)^{2}}{(a+\mathfrak{a}) q a} b^{2} \\
& =\frac{1}{2} q a^{2}+\frac{1}{2}(1+n) q\left(a^{2}-r_{2}^{2}\right)=\frac{1}{2}(2+n) q a^{2}-\frac{1}{8} \frac{(3+2 n)^{2}}{(1+n) q} b^{2} .
\end{aligned}
$$

Nun wird weiter der Inhalt der von einem beliebigen Puncte $\boldsymbol{P}$ beschriebenen Figur $\boldsymbol{W}$ gefunden, sobald man dessen Abstand $\boldsymbol{r}$, von $\boldsymbol{R}$ kennt, nämlich es ist (126) :

153.

$$
\begin{aligned}
\boldsymbol{W} & =\frac{1}{2}(2 q+q) a^{2}-\frac{1}{2}(q+\mathfrak{q}) r_{2}^{2}+\frac{1}{2}(q+q) r^{2} \\
& =\frac{1}{2} q a^{2}+\frac{1}{2}(q+q)\left(a^{2}-r_{1}^{2}+r^{2}\right) \\
& =\frac{1}{2} \frac{a+2 a}{\mathfrak{a}} q a^{2}-\frac{1}{8} \frac{(2 a+3 \mathfrak{a})^{2}}{(a+\mathfrak{a}) \mathfrak{a}^{2}} \boldsymbol{b}^{2}+\frac{1}{2} \frac{a+\mathfrak{a}}{\mathfrak{a}} q r^{2} \\
& =\frac{1}{2} q\left[(2+n) a^{2}+(1+n) r^{2}\right]-\frac{1}{8} \frac{(3+2 n)^{2}}{(1+n) q} b^{2} \\
& =\frac{1}{2} q\left[(2+n) a^{2}+(1+n) r^{2}-\frac{1}{4} \frac{(3+2 n)^{2}}{1+n}\left(\frac{b}{q}\right)^{2}\right], \text { etc. }
\end{aligned}
$$

Die Figur $\boldsymbol{W}$ ist hier ein bestimmtes Stück irgend einer Epicycloide, dessen Quadratur durch die vorstehende allgemeine Formel gegeben wird. Der Wiukel $q$ (so wie q) kann beliebig grofs sein, d. h. er kann beliebige Vielfache von $2 \pi$ enthalten, wo dann zugleich auch der Bogen $\boldsymbol{A B}$ eben so oft den ganzen Kreisumfang unfafst. Ist $q$ gerade ein Vielfaches von $2 \pi$, so ist allemal die Sehne $b=0$, und daher auch $\boldsymbol{Q R}$ oder $r_{1}=0$, d. h. daun fällt der ausgezeichnete Punct $\boldsymbol{R}$ in den Mittelpunct $\boldsymbol{Q}$ des rollenden Kreises, und aus den Formeln (152 und (153) verschwinden die mit $\boldsymbol{b}$ (oder $\boldsymbol{r}_{1}$ ) behafteten Glieder. Um dieses Verschwinden in den Formeln selbst anzudeuten, darf nur $2 a \sin \frac{1}{2} q$ statt $b$ gesetzt werden. Es sei $q=m 2 \pi$, wo $m$ eine ganze Zahl, so hat man :

$$
w=m(2+n) \pi a^{2} ; \quad W=m(2+n) \pi a^{2}+m(1+n) \pi r^{2},
$$

and wenn zugleich $\mathfrak{q}=\mathfrak{m} 2 \pi$, und $\mathfrak{m}$ ebenfalls eine ganze Zahl, jedoch $m$ und $m$ relative Primzahlen sind, so ist :

154. $w=(2 m+\mathfrak{m}) \pi a^{2}$, und

155. $W=(2 m+m) \pi a^{2}+(m+m) \pi r^{2}$,

wobei nämlich die von dem Puncte $\boldsymbol{P}$ beschriebene Curve (Epieycloide) sich schliefst (oder in sich zurückkehrt), und der Kreis $\mathfrak{B}$ oder $\boldsymbol{A B}$ gerade $m$ mal un die Basis $\mathfrak{U}$ oder $\mathfrak{A} \mathfrak{B}$ herumrollt. 
In Hinsicht der kleinsten Figur $\boldsymbol{w}$, wofern der Winkel $\boldsymbol{q}$ beliebig, wie in (158), kann noch bemerkt werdeu, dafs ihr Inhalt positiv oder negativ sein kann, und dals dazwischen $w=0$ wird, wenn :

156. $r_{1}^{2}=\frac{n+2}{n+1} a^{2}$, oder $\left(\frac{b}{q}\right)^{2}=4 \frac{(n+1)(n+2)}{(2 n+3)^{2}} a^{2}$,

und somit die Abstände $r_{1}$ und $\frac{b}{q}$ der Puncte $R$ und $\subseteq$ von dem Mittelpuncte $\boldsymbol{Q}$ des rollenden Kreises durch die Radien beider Kreise gegeben sind. Die Werthe von $W$ sind dann :

$$
\text { 157. } W=\frac{1}{2}(q+q) r^{2}=\frac{1}{2}(1+n) q r^{2} \text {. }
$$

Und wenn für diesen Fall insbesondere $a=a$, also $n=1$ ist, so hat man:

$$
\text { 158. } r_{1}^{2}=\frac{3}{2} a^{2} ; \quad\left(\frac{b}{q}\right)^{2}=\frac{24}{25} a^{2} ; \quad W=q r^{2} .
$$

Es giebt noch andere Fälle, bei allgemeineren Curven, wo der eigenthümliche Punct $\boldsymbol{R}$ sich unmittelbar angeben lärst, wie z. B. folgende.

IV. Wenn jede der beiden Curven $\mathfrak{B}, \mathfrak{U}$ geschlossen ist und die rollende $\mathfrak{B}$ einen Mittelpunct hat; wenn ferner ihre Umfänge sich verhalten, wie zwei ganze Zahlen $v: u$, die keinen gemeinschaftlichen Theiler haben, jedoch $v$ gerade ist; und wenn endlich $\mathfrak{B}$ so lange rollt, bis sie wieder genau in ihre anfüngliche Lage gelangt, d. h. bis wieder die nëmlichen Puncte $A$ und $\mathfrak{A}$ beider Curven sich treffen, was erst nach $v$ Umläufen der $\mathfrak{B}$ um $\mathfrak{U}$ eintritt, und wo dann jeder mit $\mathfrak{B}$ verbundene Punct $\boldsymbol{P}$ in seine ursprüngliche Lage kommt, also die von ihm beschriebene Curve $W$ in sich zurückkehrt: so fältt der eigenthünliche Punct $\boldsymbol{R}$ allemal mit dem Mittelpuncte der rollenden Curve $\mathfrak{B}$ zusammen.

Nämlich unter diesen Bedingungen vereinigen sich die vier Puncte $\boldsymbol{S}, \boldsymbol{S}_{1}, \mathcal{S}$ und $\boldsymbol{R}$ alle nit dem Mittelpuncte der Curve $\mathfrak{B}$. Deun dafs zunächst $S$ in denselben falle, ergiebt sich darans, dafs der in Betracht kommende Bogen $\boldsymbol{A B}$ bei $\mathfrak{B}$ gerade aus dem $\boldsymbol{u}$ fachen Umfange dieser Curve besteht, folglich der Krümmungs-Schwerpunct $\boldsymbol{S}$ des ganzen Bogens mit dem des einfachen Umfanges der Curve $\mathfrak{B}$ zusammenfält und mithin der Mittelpunct der letztern ist (\$. XXII.). Zugleich folgt hieraus, dafs der Winkel $g=u \cdot 2 \pi$, und da der Endpunct $B$ des Bogens mit dem Anfangspuncte $A$ zusammenfält, dafs die Sehne $b=0$ ist. Eben so ist der Winkel $\mathfrak{q}=v .2 \pi$, weil der überrollte Bogen $\mathfrak{2} \mathfrak{B}$ aus dem $v$ fachen $\mathrm{Um}-$ ringe der Basis $\mathfrak{U}$ besteht. 
Um zu zeigen, dafs auch der Schwerpunct $S_{1}$ des Bogens $A B$, welcher von der Krümmung der Basis $\mathfrak{A B}$ abhängt, in denselben Mittelpunct falle, denke man die Curven $\mathfrak{B}$ und $\mathfrak{U}$, von den Anfangspuncten $A$ und $\mathfrak{A}$ aus, beziehlich in $v$ und $u$ gleiche Theile getheilt; so sind diese Theile alle von gleicher Länge. Die Theile von $\mathfrak{O}$ mögen nach der Reihe, von $A$ anfangend, durch $\mathfrak{B}_{1}, \mathfrak{B}_{2}, \mathfrak{B}_{3}, \ldots \mathfrak{B}_{v}$ bezeichnet werden. Sie stehen einander paarweise gegenüber und sind congruent - weil $\mathfrak{B}$ einen Mittelpunct hat und $v=2 n$ eine gerade $Z$ ahl ist - so dafs also $\mathfrak{B}_{1}=\mathfrak{B}_{n+1}$, $\mathfrak{B}_{2}=\mathfrak{B}_{n+2}, \ldots \mathfrak{V}_{n}=\mathfrak{B}_{2 n}$, und dafs ferner irgend ein Punct $\boldsymbol{X}_{1}$ in $\mathfrak{B}_{\ell}$ und der homologe Punct $\boldsymbol{X}_{n+1}$ in $\mathfrak{B}_{n+1}$ allemal die Endpuncte eines Durchmessers der Curve $\mathfrak{B}$ sind, also ihr Mittelpunct in der Mitte der Geraden $\boldsymbol{X}_{1} \boldsymbol{X}_{n+1}$ liegt. Heifsen die Theile der Basis $\mathfrak{U}$, von $\mathfrak{A}$ aus nach entsprechender Richtung genommen, $\mathfrak{u}_{1}, \mathfrak{U}_{2}, \mathfrak{u}_{3}, \ldots \mathfrak{U}_{u}$. Jeder dieser Theile wird je einmal von jedem der $v$ Umfangstheile der $\mathfrak{B}$ - während diese $v$ Unläufe um $\mathfrak{U}$ macht - überrollt, wovon man sich durch blofses $A b$ zählen leicht überzeugt. In irgend einem Theile von $\mathfrak{U}$, etwa in $\mathfrak{U}_{x}$, fixire man einen beliebigen Punct $\mathfrak{X}$ : so kommt derselbe mit solchen $v$ Puncten $\boldsymbol{X}_{1}, \boldsymbol{X}_{2}, \boldsymbol{X}_{3}, \ldots \boldsymbol{X}_{2 n}$ der rollenden $\mathfrak{V}$ in Berührung, welche auf ihre $v \mathrm{U}_{\mathrm{m}}$ fangstheile $\mathfrak{V}_{1}, \mathfrak{V}_{2}, \ldots \mathfrak{V}_{2 n}$ so vertheilt, dafs sie die Endpuncte von $n$ Durchmessern der $\mathfrak{B}$ sind. Daher haben die Gewichte, welche je einem System von solchen $v$ Puncten $X_{1}, X_{2}, \ldots X_{2 n}$, vermöge der Krümmung der Basis $\mathfrak{U}$ im Puncte $\mathfrak{X}$, zukommen, allemal den Mittelpunct der Curve $\mathfrak{Z}$ zum Schwerpunct; und folglich mufs auch der gemeinschafliche Schwerpunct aller Systeme, d. i. $\boldsymbol{S}_{1}$, in diesen Mittelpunct fallen.

Wemn aber $\boldsymbol{S}$ und $\boldsymbol{S}_{1}$ zusammenfallen, so vereinigt sich auch $\mathfrak{S}$ mit ihnen; und da ferner die Sehne $b=0$ ist, so liegt auch $\boldsymbol{R}$ im nämlichen Puncte, so dafs also die vier Puncte $S, S_{1}, \subseteq$ und $R$ alle mit dem Mittelpuncte der rollenden Curve $\mathfrak{B}$ zusammenfallen.

Werden die oben angezeigten Werthe für die Winkel $q$ und $q$ in die Formel (126) gesetzt, so bat man für den gegenwärtigen Fall:

das heifst :

159. $W=w+(v+u) \pi r^{2}$,

„Wird in einer beliebigen Kreislinie, welche mit der rollenden Curve $\mathfrak{B}$ denselben Mittelpunct $R$ hat, irgend ein Punct $P$ angenommen, so ist die von ihm beschriebene Figur $W$ allemal gerade um die $v+u$ fache Kreisflüche grö/ser, als die vom Mittelpuncte $R$ beschriebene Fügur w." 
In Rücksicht der obigen Bedingungen (IV.) kann man verschiedene Modificationen eintreten lassen, wobei dann analoge Resultate statt finden, wie z. B.

1. „Wenn $\mathfrak{Y}$ insbesondere ein Kreis, dagegen die Zahl $v$ beliebig - gerade oder ungerade - nur nicht $=1$ *) und wenn immerhin $v$ und $u$ relative Primzahlen sind: so findet der Satz gleicherweise statt."

Denn wenn auch $v$ ungerade ist, so haben doch die Puncte $\boldsymbol{X}_{1}$, $\boldsymbol{X}_{2}, \boldsymbol{X}_{3}, \ldots \boldsymbol{X}_{v}$, da sie nothwendig den Umfang des Kreises $\mathfrak{B}$ in $(v)$ gleiche Theile theilen, immerhin dessen Mittelpunct zum Schwerpunct.

Für diesen Fall hat man, wenn der Radius des Kreises $=a$ gesetzt wird (150) :

$$
\begin{aligned}
& \text { 160. } w=(2 v+u) \pi a^{2} \text {, und } \\
& \text { 161. } W=(2 v+u) \pi a^{2}+(v+u) \pi r^{2} \text {, }
\end{aligned}
$$

und für den speciellen Fall, wo $\boldsymbol{P}$ in $\operatorname{der}$ Kreislinie $\mathfrak{B}$ selbst liegt:

$$
\text { 162. } W=(3 v+2 u) \pi a^{2} \text {. }
$$

In Hinsicht dieser Formeln, so wie in Bezug auf (159), ist zu bemerken : „da/s die nähere Form der Basis $\mathfrak{U}$, wofern nur ihr Umfang den geforderten Bedingungen genügt, auf den Inhalt der Fïguren $W$ und u, keinen Einflu/s hat." Eben so verhält es sich bei einigen früheren Formeln.

2. „Wenn $\mathfrak{B}$ beschaffen ist wie anfangs (IV.), dagegen $\mathfrak{U}$ auch einen Mittelpunct hat, und wenn die Zahlen $v$ und $u$ beide ungerade - aber immerhin relative Primzahlen - sind: so findet der Satz, sammt der Formel (159), gleicherweise statt."

Denn wenn $\mathfrak{U}$ einen Mittelpunct hat, so hat sie in den Endpuncten $\mathfrak{X}, \mathfrak{Y}$ jedes Durchmessers gleiche Krümmung; zwei solche Puncte aber treffen mit zwei Reihen Puncten auf $\mathfrak{B}$ zusammen, etwa mit $\boldsymbol{X}_{1}, \boldsymbol{X}_{2}, \ldots$ $\ldots \boldsymbol{X}_{v}$ und $\boldsymbol{Y}_{1}, \boldsymbol{Y}_{2}, \ldots \boldsymbol{Y}_{v}$, welche paarweise die Endpuncte von Durchmessern der $\mathfrak{B}$ sind, nämlich so gepaart, dafs je ein Punct $\boldsymbol{X}$ mit irgend einem Puncte $\boldsymbol{Y}$ zusammengehört, (weil $\boldsymbol{v}$ und $\boldsymbol{u}$ ungerade sind); daher mufs der Schwerpunct dieser zwei Reihen Puncte, wenn sie - vermöge der Krümmungen in $\mathfrak{X}$ und $\mathfrak{Y}$ - gleiche Gewichte haben, in den Mittelpunct der Curve $\mathfrak{B}$ fallen; woraus folgt, dafs auch der Schwerpunct $\boldsymbol{S}_{\mathbf{1}}$ in denselben Mittelpunct fällt.

Dieser Satz findet auch statt, wenn insbesondere $v=u=1$.

*) Diese Bedingung wurde durch ein Versehen bei der ersten Mittheilung des Satzes (in diesem Journal Bd. 18. S. 278) nicht ganz richtig angegeben. 


\section{\$. XXXVI.}

Zum Schlusse füge ich noch folgende Bemerkungen hinzu.

1. Wenn insbesondere die beiden Curven $\mathfrak{B}$ und $\mathfrak{U}$ einander gleich sind (congruent) und wenn sie einander - während $\mathfrak{B}$ auf $\mathfrak{U}$ rollt - stets in homologen Puncten berühren, so ist die von irgend einem mit $\mathfrak{B}$ verbundenen Puncte $\boldsymbol{P}$ beschriebene Curve $\boldsymbol{W}$ allemal der dem homologen Puncte $\mathfrak{P}$ in Bezug auf die Basis $\mathfrak{U}$ entsprechenden Fufspuncten-Curve $\boldsymbol{V}$ älnlich, und zwar haben dieselben den festen Punct $\mathfrak{P}$ zum (äufsern) Aehnlichkeitspunct und ihre entsprechenden Dimensionen verhalten sich, wie $2: 1$. Denn die gemeinschaftliche Tangente der Curven $\mathfrak{B}$ und $\mathfrak{U}$ in ihrem Berührungspuncte $(\boldsymbol{A} \mathfrak{\mathfrak { I }})$ geht offeubar in jedem Augenblicke durch die Mitte der Geraden $\mathfrak{P P}$ und steht auf ihr senkrecht; woraus das Behauptete folgt.

Zugleich folgt hieraus, dafs die Curve $W$ selbst als FufspunctenCurve angesehen werden kann, nämlich des Punctes $\mathfrak{P}$ in Bezug auf eine Curve $\mathfrak{U}_{1}$, welche der $\mathfrak{U}$ ähnlich, mit ihr $\mathfrak{P}$ zum Aelhnlichkeitspunct und zudem doppelt so grofse Dimensionen, als diese, hat. So z. B. sind also die sämmtlichen Fufspuncten-Curven in Bezug auf einen gegebenen Kreis nichts anderes, als die verschiedenen Epicycloiden, welche entstehen, wenn der rollende Kreis der Basis gleich, und wenn ihr Durchmesser dem Radius jenes Kreises gleich ist. Gleiche Folgerungen ergeben sich für die übrigeu Kegelschnitte; woraus verschiedene Sätze hervorgehen, deren nähere Angabe hier übergangen wird *).

Ueberhaupt finden also hier für die Figuren $W$ die nänlichen Gesetze statt, wie oben für die Fufspuncten-Figuren $V$ (Anm. \$. XXXV. I, 1. und §. XXI.); denn immer fällt der Punct $\boldsymbol{S}_{1}$ - und somit auch $\mathfrak{S}-$ mit dem Krümmungs-Schwerpuncte $\boldsymbol{S}$ zusammen, und der nämliche Punct $\boldsymbol{R}$, welchem die kleinste Fufspuncten-Figur $v$ entspricht, beschreibt auch die kleinste Figur $\boldsymbol{w}$.

2. Ist $\boldsymbol{A B}$ Bogen eines Kreises $\mathfrak{B}$, dessen Radius $=a$, und $\mathfrak{A} \mathfrak{B}$ eine beliebige, stetig convexe Curve, auf deren convexen Seite $\boldsymbol{A B}$ rollt; sind ferner $\boldsymbol{P}_{1}, \boldsymbol{P}_{2}, \boldsymbol{P}_{3}, \ldots \boldsymbol{P}_{n}$ irgend ein System von $\boldsymbol{n}$ Puncten in der Ebene des Kreises die dessen Mittelpunct $\boldsymbol{Q}$ zum Schwerpuncte haben und von

*) Einige von diesen Sätzen befinden sich in Klügel's Math. Wörterb. Art. Epicykloide, wo es aber (Bd. II. S. 128) statt : der ,Durchmesser" der von den Brennpuncten beschriebenen Kreise sei gleich der Hauptaxe der Ellipse oder Hyperbel; heifsen mufs : der "Radius" etc. 
ihm beziehlich um $r_{1}, r_{2}, r_{3}, \ldots r_{n}$ abstehen; wird $r_{1}^{2}+r_{2}^{2}+r_{3}^{2}+\ldots .+r_{n}^{2}$ $=s^{2}$ gesetzt, und eben so die Summe der von den $n$ Puncten beschriebenen Figuren $W_{1}, W_{2}, \ldots W_{n}$ durch $S$, so wie die Summe der $n$ excentrischen Kreissectoren $\boldsymbol{P}_{1} \boldsymbol{A B}, \boldsymbol{P}_{2} \boldsymbol{A B}, \ldots . \boldsymbol{P}_{n} \boldsymbol{A B}$ durch $\mathfrak{S}$ bezeichnet, so hat man :

$$
\text { 163. } S=\mathfrak{S}+\frac{1}{2} n(q+\mathfrak{q}) \cdot a^{2}+\frac{1}{2}(q+\mathfrak{q}) \cdot s^{2} \text {. }
$$

Liegen die $n$ Puncte $\boldsymbol{P}_{1}, \boldsymbol{P}_{2}, \ldots$ in einer mit $\mathfrak{B}$ concentrischen Kreislinie, deren Radius $=r$, so ist :

$$
\text { 164. } S=\mathfrak{S}+\frac{1}{2} n(q+q) \cdot\left(a^{2}+r^{2}\right) \text {. }
$$

Ist die Curve $\mathfrak{A} \mathfrak{B}$ oder $\mathfrak{U}$ geschlossen, verhalten sich die Umfänge von $\mathfrak{B}$ und $\mathfrak{U}$ wie zwei relative Primzahlen $v$ und $u$, und rollt $\mathfrak{B}$ gerade $\boldsymbol{v}$ mal um $\mathfrak{U}$ herum, wo dann die $\boldsymbol{n}$ Puncte in ihre anfängliche Lage zurückkehren und $q=u .2 \pi, \mathfrak{q}=v .2 \pi$ wird, so ist jeder Sector $=u . \pi a^{2}$ und man hat (163 u. 164):

165. $S=n u \cdot \pi a^{2}+n(u+v) \cdot \pi a^{2}+(u+v) \cdot \pi s^{2}$, und

166. $S=n(2 u+v) \cdot \pi a^{2}+n(u+v) \cdot \pi r^{2}$.

Haben $\mathfrak{B}$ und $\mathfrak{U}$ gleichen Umfang, so dafs $v=u=1$, so ist beziehlich :

$$
\begin{aligned}
& \text { 167. } S=3 n . \pi a^{2}+2 \pi s^{2} \text {, und } \\
& \text { 168. } S=3 n . \pi a^{2}+2 n \cdot \pi r^{2} \text {, }
\end{aligned}
$$

und wenn $r=a$, also die $n$ Puncte in der Kreislinie $\mathfrak{B}$ selbst liegen, so ist : 169. $S=5 n \cdot \pi a^{2}$.

Hat die Basis $\mathfrak{U}$ einen Mittelpunct, so haben die Figuren $W_{1}$, $W_{2}, \ldots W_{n}$, in jedem der zwei letzteren Fälle (168 u. 169), unter sich gleichen Inhalt, so dafs also für jede einzeln, beziehlich :

$$
\begin{array}{ll}
\text { 170. } & W=3 \pi a^{2}+2 \pi r^{2} \text {, und } \\
\text { 171. } & W=5 \pi a^{2} \text {. }
\end{array}
$$

Wie man sieht, sind auch die vorstehenden Formeln von der speciellen Natur der Basis $\mathfrak{U}$ (ihrer Gleichung etc.) unabhängig (s. \$. XXXV. IV. 1.).

Mehrere von den in dieser Abhandlung vorgetragenen Sätzen habe ich bereits früher in diesem Journal Bd. XVIII. zu beweisen vorgelegt. 